\title{
Spaces for agreement: a theory of Time-Stochastic Dominance and an application to climate change
}

\author{
Simon Dietz* and Nicoleta Anca Matei ${ }^{\dagger}$
}

December 22, 2014

\begin{abstract}
Many investments involve both a long time-horizon and risky returns. Making investment decisions thus requires assumptions about time and risk preferences. Such assumptions are frequently contested, particularly in the public sector, and there is no immediate prospect of universal agreement. Motivated by these observations, we develop a theory and method of finding 'spaces for agreement'. These are combinations of classes of discount and utility function, for which one investment dominates another (or 'almost' does so), so that all decision-makers whose preferences can be represented by such combinations would agree on the option to be chosen. The theory is built on combining the insights of stochastic dominance on the one hand, and time dominance on the other, thus offering a nonparametric approach to inter-temporal, risky choice. We go on to apply the theory to the controversy over climate policy evaluation and show with the help of a popular simulation model that, in fact, even tough carbon emissions targets would be chosen by almost everyone, barring those with arguably 'extreme' preferences.
\end{abstract}

Keywords: almost stochastic dominance, climate change, discounting, integrated assessment, project appraisal, risk aversion, stochastic dominance, time dominance, time-stochastic dominance

JEL codes: D61, H43, Q54

${ }^{*}$ Grantham Research Institute on Climate Change and the Environment, ESRC Centre for Climate Change Economics and Policy, and Department of Geography and Environment, London School of Economics and Political Science.

${ }^{\dagger}$ European Commission, Joint Research Centre (JRC), Institute for Prospective Technological Studies (JRC-IPTS), Seville.

The views expressed are purely those of the authors and may not in any circumstances be regarded as stating an official position of the European Commission. 
We would like to acknowledge very constructive comments from the editor and two anonymous referees. We have also benefitted from the input of Alec Morton, Claudio Zoli, and seminar participants at AARES 2014, the Global-IQ meetings in Brussels and Rome, the Graduate Institute Geneva, Hamburg University, LSE, Manchester University and WCERE 2014. This research has received funding from the European Union's Seventh Framework Programme (FP7/2007-2013) under grant agreement 266992 - Global-IQ "Impacts Quantification of Global Changes". The usual disclaimer applies.

Email for correspondence: s.dietz@lse.ac.uk

\section{Introduction}

When making investment decisions one is frequently confronted with long timehorizons and risky returns. Therefore assumptions about time and risk preferences are important. Making such assumptions is always tricky. In the area of public project appraisal they are especially contested, because, on top of the usual challenges of estimating individual preferences, there are positions to be taken on how to aggregate individual preferences into social preferences.

A particularly good example of a long-run, risky public investment is climatechange mitigation. It comes as no surprise then that great controversy surrounds policy proposals to abate greenhouse gas emissions, and that this controversy has turned in large measure on positions taken on time and risk preferences. By now the debate will be familiar to readers, so a very short summary might suffice here.

In the context of a model where social welfare is the discounted sum of individual utilities, the pioneering studies of Cline (1992) and Nordhaus (1991; 1994) staked out debating positions on pure time preference that still hold today - Cline set the utility discount rate to $0 \%$ based on so-called 'prescriptive' ethical reasoning, while Nordhaus set it to $3 \%$ based on a more conventional 'descriptive' analysis of market rates of investment returns. ${ }^{1}$ More recently, the Stern Review (Stern, 2007) set the utility discount rate to $0.1 \%$ and advocated aggressive emissions abatement, with the former assumption seemingly causing the latter result. ${ }^{2}$ However, the Stern Review also prompted debate about the appropriate utility function, which in the standard model simultaneously represents risk preferences and preferences to smooth consumption over time. Questions have included the appropriate degree of risk/inequality aversion in an iso-elastic function (e.g. Dasgupta, 2007; Gollier, 2006; Stern, 2008), and the appropriate function itself (Ikefuji et al., 2012; Pindyck, 2011).

Rather than attempting to settle the debate, in this paper we embrace it. Our starting point is the supposition that debate about time and risk preferences legitimately exists and will endure. Given the ingredients of the debate and the

\footnotetext{
${ }^{1}$ See Arrow et al. (1996) for a classic comparison of these two points of view, from where the labels descriptive and prescriptive hail.

${ }^{2}$ See Nordhaus $(2007 ; 2008)$ for critiques of the Stern Review.
} 
current state of knowledge, "reasonable minds may differ" (Hepburn and Beckerman, 2007). Why is the debate difficult to resolve? It contains normative and positive elements. There is a clear sense in which normative differences may never be completely eliminated. Positive 'uncertainties' could in principle be eliminated by collecting more empirical data from, for instance, market behaviour, questionnaire surveys or laboratory experiments, but in reality it is likely that they will also persist (as with longstanding puzzles in the economics of risk, such as the equity premium and risk-free rate).

Consequently we are in the search for partial rather than complete orderings of choices. We want to establish a theory and method of identifying whether there exist 'spaces for agreement', that is combinations of classes of discount and utility function, for which one investment dominates another (or 'almost' does so), so that all decision-makers whose preferences can be represented by such combinations would agree on the option to be chosen.

Why might this be useful? Given disagreement about appropriate time and risk preferences, our approach does not require decision-makers to make $a$ priori choices of functional form or parameter values. While this non-parametric approach could be used to inform investment choice in the private sector, its main use is more likely to be to bring renewed clarity to hotly contested choices in public policy, such as mitigation of climate change. In these areas, the debate about time and risk preferences might have become a distraction, preventing us from asking whether in fact there are some meaningful courses of action that both sides could agree to take.

The intellectual antecedents of this paper lie in the theory of Stochastic Dominance (Fishburn, 1964; Hanoch and Levy, 1969; Hadar and Russell, 1969; Rothschild and Stiglitz, 1970) and its offshoots, in particular Almost Stochastic Dominance (Leshno and Levy, 2002), Time Dominance (Bøhren and Hansen, 1980; Ekern, 1981) and extensions of dominance analysis to multivariate problems (Levy and Paroush, 1974b; Atkinson and Bourguignon, 1982; Karcher et al., 1995).

Stochastic Dominance (SD) is a fundament of the theory of decision-making under uncertainty. It is undoubtedly useful for the sort of problems we have just set out, precisely because it offers a non-parametric approach to risky choice, whereby one tests for SD relations for whole preference classes. However, the basic theory of SD is a-temporal. In effect, decisions are made and pay-offs obtained in the same time period. While extensions have been made to the multiperiod case (Levy, 1973; Levy and Paroush, 1974a), the decision-maker is not permitted to prefer flows of utility in some periods of time more than in others. $^{3}$ This is a serious drawback, as it is clear that most decision-makers are impatient, preferring utility now to utility later on. Time preference is, by contrast, the core focus of the theory of Time Dominance (Bøhren and Hansen, 1980; Ekern, 1981), which takes the SD machinery and applies it to cashflows, i.e. instead of working with cumulative distributions over the consequence space

\footnotetext{
${ }^{3}$ One exception we are aware of is Scarsini (1986), who looked at a special case of utility discounting. We will clarify the relationship between his paper and ours later.
} 
of a decision, one works with cumulative distributions over time. Like SD, one tests for a Time Dominance (TD) relation for whole preference classes, rather than having to pre-specify and parameterise a discount function. The drawback of TD, however, is the obverse of SD, namely that the basic theory has been developed for certain, rather than uncertain, cashflows, and can only be extended to the latter under restrictive assumptions (see Annex 1).

Another drawback of the basic theory of SD is nicely illustrated by a stylised example from Levy (2009) - try to use SD criteria to rank two prospects, one of which pays out $\$ 0.5$ with a probability of 0.01 and $\$ 1$ million with a probability of 0.99 , and the other of which pays out $\$ 1$ for sure. While it would seem that virtually any investor would prefer the former, SD cannot be established. ${ }^{4}$ Arguably this paradox betrays the disadvantage of SD's generality - within the classes of utility function considered, there are some 'extreme' (Leshno and Levy, 2002) or even 'pathological' (Levy, 2009) utility functions, according to which the latter prospect is preferred. ${ }^{5}$ For this reason Leshno and Levy (2002) derived Almost Stochastic Dominance (Almost SD), according to which one compares the area between the cumulative distributions in which SD is violated with the total area between the distributions. Crucially, the ratio of the former to the latter can be given an interpretation in terms of restrictions on the class of utility functions, and if the restriction is very small, an Almost SD relation can be argued to exist.

This sets the conceptual task for the present paper, which is to unify the theories of SD and TD so that we have at our disposal a general framework for choosing between risky, inter-temporal prospects, which admits the possibility of pure-time discounting and makes weak assumptions about the risk characteristics of the prospects: Time-Stochastic Dominance (TSD). In addition, we extend the notion of Almost SD to our bi-dimensional time-risk setup, defining Almost TSD. This provides a way to exclude extreme combinations of time and risk preferences and promises to greatly increase the practical usefulness of the framework.

We then make an empirical application of the theory to climate change, by analysing a set of trajectories for global greenhouse gas emissions - a set of 'policies' - using a stochastic version of the benchmark DICE integrated assessment model devised by Nordhaus. ${ }^{6}$ Our results show the climate-change debate in a new light. Although the profile of net benefits from climate mitigation is such that 'exact' TSD cannot be established, the less restrictive concept of Almost TSD allows us to show that the space for agreement on climate change

\footnotetext{
${ }^{4}$ Where $F^{n}(x)$ and $G^{n}(x)$ are respectively the $\mathrm{n}^{\text {th }}$-order cumulative distributions of the former and latter prospects over realisations $x$, this is because the first nonzero values of $G^{n}(x)-F^{n}(x)$ are negative as $x$ increases from its lower bound. However, $\mathrm{n}^{\text {th }}$-order SD requires that $G^{n}(x)-F^{n}(x) \geq 0, \forall x, E_{F}(x) \geq E_{G}(x)$ and there is at least one strict inequality. See Annex 1 for further explanation.

${ }^{5}$ In the example used, one would be $u(x)=\left\{\begin{array}{c}x \text { for } x \leq 1 \\ 1 \text { for } x>1\end{array}\right.$.

${ }^{6}$ The first version appeared in Nordhaus (1993b; 1993a). We build on the version in Nordhaus (2008).
} 
is indeed large. Since Almost TSD is based on the notion of excluding extreme combinations of time and risk preferences, this result in particular lends itself to the following rather stark interpretation: only those with 'extreme' preferences over time and risk would prefer not to cut carbon emissions by a large amount.

The remainder of the paper is set out as follows. In the next short section we deal with some analytical preliminaries, in particular we set out the classes of utility and discount function that will be of primary focus. In Section 3 we establish the theory of (exact) TSD, while in Section 4 we do the same for Almost TSD. Section 5 describes how we set up the DICE model, while Section 6 presents our results and Section 7 concludes.

\section{Spaces for agreement}

Readers interested in quickly getting up to speed with the existing literatures on SD and TD theory are referred to the short primer in Annex 1. Building on this, let us take the task at hand as being to rank two prospects $X$ and $Y$, both of which yield random cashflows over time. The underlying purpose is to compare the expected discounted utilities of the prospects at $t=0$, i.e. for prospect $X$ we compute

$$
N P V_{v, u}(X) \equiv \int_{0}^{T} v(t) E_{F} u[x(t)] d t=\int_{0}^{T} v(t)\left[\int_{a}^{b} u(x) f(x, t) d x\right] d t
$$

where $x$ is a realisation of the cashflow of prospect $X, v$ is a discount function and $u$ is a utility function. Both functions $v$ and $u$ are assumed to be continuous and continuously differentiable at least once. We make the assumptions, characteristic in the dominance literature, that the random cashflows of $X$ and $Y$ are both supported on the finite interval $[a, b]$, and that each prospect pays out over a finite, continuous time-horizon $[0, T]$. Therefore we can characterise a probability density function for prospect $X$ at time $t \in[0, T], f(x, t)$, and a counterpart cumulative distribution function with respect to realisation $x \in[a, b]$ at time $t \in[0, T], F^{1}(x, t)=\int_{a}^{x} f(s, t) d s$. Note that because utility is additively separable across time in (1), no particular assumption is required about the serial correlation of the probability distribution.

Before characterising Time-Stochastic Dominance (TSD), we need to define classes of utility and discount functions. Our broadest class of utility function $u:[a, b] \rightarrow \mathbb{R}$ is $U_{1}=\left\{u: u^{\prime}(x) \geq 0\right\}$, i.e. the class of utility functions, whereby utility is non-decreasing as a function of consumption, representing nothing more than (weak) non-satiation. It is hard to imagine relevant circumstances in which the appropriate utility function would not be in $U_{1}$. More generally, any subset $m$ of utility functions is defined recursively as

$$
U_{m}=\left\{u: u \in U_{m-1} \text { and }(-1)^{m} u^{m}(x) \leq 0\right\} .
$$

where, among other things, $m$ represents the number of times that $u(x)$ is differentiated. As well as $U_{1}$, in this paper we focus on $U_{2}=\left\{u: u \in U_{1}\right.$ and $\left.u^{\prime \prime}(x) \leq 0\right\}$, 
which is the class of non-decreasing, weakly concave utility functions, ruling out risk-seeking. Whether the appropriate utility function is in $U_{2}$ is a little less clear, but it is almost certainly a good description of most individual behaviour, and there are few if any arguments for public policy evaluation to be based on risk-seeking. Eventually we establish a theorem for TSD of an arbitrarily high order with respect to both time and risk.

Let us define a corresponding set of discount functions on the time domain, $v:[0, T] \rightarrow \mathbb{R}$. The broadest class of discount functions requires simply that at any point in time more is preferred to less, $V_{0}=\{v: v(t)>0\}$. However, $V_{0}$ is of little interest, since some positive degree of time preference is always required, however small. More generally, any subset $n$ of discount functions is defined recursively as

$$
V_{n}=\left\{v: v \in V_{n-1} \text { and }(-1)^{n} v^{n}(t)>0\right\} .
$$

Our attentions will be focused on $V_{1}=\left\{v: v \in V_{0}\right.$, and $\left.v^{\prime}(t)<0\right\}$, the class of strictly decreasing discount functions, exhibiting positive time preference. We could proceed to $V_{2}=\left\{v: v \in V_{1}\right.$, and $\left.v^{\prime \prime}(t)>0\right\}$, the class of strictly decreasing, convex discount functions, but since both $V_{1}$ and $V_{2}$ admit the most popular exponential and hyperbolic discount functions as special cases, there is little to gain from doing so.

Combinations of these classes of utility and discount functions constitute possible spaces for agreement. $V_{1} \times U_{1}$ is the largest possible space for agreement that we consider, encapsulating any decision-maker whose preferences can be represented by, respectively, a strictly decreasing discount function and a nondecreasing utility function, in other words any impatient decision-maker with any attitude to risk. Presumably virtually all decision-makers belong to this combination of classes. By contrast $V_{1} \times U_{2}$, for instance, encapsulates any impatient decision-maker who is not risk-seeking. Whether there is an actual space for agreement depends of course on whether any dominance relations can be established between projects, for the combination in question. Note that in Section 4 we narrow these spaces for agreement further by placing additional restrictions on $V$ and $U$ with a view to excluding 'extreme' combinations of time and risk preferences.

\section{Time-Stochastic Dominance}

A further piece of notational apparatus will enable us to work in a compact, bi-dimensional form. Denote the integral over time of the $p d f$ by $F_{1}(x, t)=$ $\int_{0}^{t} f(x, w) d w$, while the integral over time of the $c d f$ is

$$
F_{1}^{1}(x, t)=\int_{a}^{x} F_{1}(s, t) d s=\int_{0}^{t} F^{1}(x, w) d w=\int_{0}^{t} \int_{a}^{x} f(s, w) d s d w
$$

Defining $d(z, t)=g(y, t)-f(x, t)$, we set

$$
D_{i}^{j}(z, t)=G_{i}^{j}(y, t)-F_{i}^{j}(x, t)
$$


for all $x, y, z \in[a, b]$ and all $t \in[0, T]$. Given information on the first $n$ and $m$ derivatives of the discount and utility functions respectively, we recursively define:

$$
\begin{gathered}
D_{n}(z, t)=\int_{0}^{t} D_{n-1}(z, w) d w \\
D^{m}(z, t)=\int_{a}^{z} D^{m-1}(s, t) d s \\
D_{n}^{m}(z, t)=\int_{0}^{t} D_{n-1}^{m}(z, w) d w=\int_{a}^{z} D_{n}^{m-1}(s, t) d s=\int_{0}^{t} \int_{a}^{z} D_{n-1}^{m-1}(s, w) d s d w
\end{gathered}
$$

where $i \in\{1,2, \ldots, n\}$ is the order of TD (i.e. the number of integrations with respect to time) and $j \in\{1,2, \ldots, m\}$ is the order of SD (i.e. the number of integrations with respect to the probability distribution). Note that our concept of TD relates to pure time discounting, whereas standard TD relates to discounting of consumption.

With all of our notation now set out, let us characterise TSD for various combinations of classes of $U_{j}$ and $V_{i}$.

Definition 1. [Time-Stochastic Dominance of order $i, j]$ For any two risky, inter-temporal prospects $X$ and $Y$

$$
X>_{i T j S} Y \text { if and only if } \Delta \equiv N P V_{v, u}(X)-N P V_{v, u}(Y) \geq 0,
$$

for all $(v, u) \in V_{i} \times U_{j}$.

In this definition, the ordering $>_{i T j S}$ denotes pure TD of the $i^{t h}$ order, combined with SD of the $j^{\text {th }}$ order. For example, $>_{1 T 1 S}$, which we can shorten to $>_{1 T S}$, denotes pure-time and stochastic dominance of the first order.

Proposition 1. [First-order Time-Stochastic Dominance] $X>_{1 T S} Y$ if and only if

$$
D_{1}^{1}(z, t) \geq 0, \forall z \in[a, b] \text { and } \forall t \in[0, T],
$$

and there is a strict inequality for some $(z, t)$.

Proof. See Annex 2.

Proposition 1 tells us that any impatient planner with monotonic nondecreasing preferences will prefer prospect $X$ to prospect $Y$, provided the integral over time of the $c d f$ of $Y$ is at least as large as the integral over time of the $c d f$ of $X$, for all wealth levels and all time-periods, and is strictly larger somewhere. It maps out a space for agreement, as we can say that all decisionmakers with preferences that can be represented by $V_{1} \times U_{1}$ will rank $X$ higher than $Y$, no matter what precisely is their discount function or utility function within these classes. ${ }^{7}$

Consider the following stylised example, comprising discrete cashflow distributions in discrete time. The use of discrete data makes the exposition easy, moreover it is also the form of data that would typically be encountered in practical applications; for instance the output of the DICE climate-change model is

\footnotetext{
${ }^{7}$ Proposition 1 is similar to Theorem 3 in Scarsini (1986). However Scarsini did not consider any other cases, i.e. any other combinations of time and risk preference.
} 
in just this form. However, it means that we have to relate Proposition 1 , stated in terms of cumulative distribution functions, to a parallel theorem stated in terms of quantile distribution functions.

Example 1. Consider prospects $X$ and $Y$, each of which comprises a cashflow over five periods of time and in four states of nature with equal probability (i.e. uniform discrete distributed):

\begin{tabular}{cc|ccccc}
\hline \multirow{2}{*}{ Prospect } & Probability & 0 & Time & period & & \\
\hline \multirow{3}{*}{$X$} & $1 / 4$ & -2 & -3 & 2 & 2 & 1 \\
& $1 / 4$ & -1 & -2 & -2 & 3 & 1 \\
& $1 / 4$ & 0 & -2 & -2 & 5 & 6 \\
& $1 / 4$ & 0 & 0 & -2 & 4 & 2 \\
\hline \multirow{3}{*}{$Y$} & $1 / 4$ & -5 & -3 & 2 & 3 & 7 \\
& $1 / 4$ & -4 & -3 & 2 & 3 & 1 \\
& $1 / 4$ & -4 & -1 & -1 & 0 & 1 \\
& $1 / 4$ & -4 & 0 & 1 & 1 & 6 \\
\hline
\end{tabular}

Instead of integration with respect to time, we simply use summation. For each additional restriction placed on the curvature of the discount function, a new round of summation of the cashflows is performed, $X_{n}(t)=\sum_{w=0}^{t} X_{n-1}(w)$. Matters on the stochastic dimension are a little more involved: we extend the quantile approach of Levy and Hanoch (1970) and Levy and Kroll (1979). Take $X$ to be an integrable random variable with, for each $t \in[0, T]$, a $c d f F^{1}(x, t)$ and an $r$-quantile function $F^{-1, r}(p, t)$, the latter of which is recursively defined as

$$
\begin{aligned}
& F^{-1,1}(p, t): \quad=\inf \left\{x: F^{1}(x, t) \geq p(t)\right\}, \forall t \in[0, T] \\
& F^{-1, r}(p, t): \quad=\int_{0}^{p} F^{-1,1}(y, t) d y, \forall p \in[0,1], \forall t \text { and } r \geq 2 .
\end{aligned}
$$

Proposition 2. (1TSD for quantile distributions). $X>_{1 T S} Y$ if and only if

$$
H_{1}^{-1,1}(p, t)=F_{1}^{-1,1}(p, t)-G_{1}^{-1,1}(p, t) \geq 0, \forall p \in[0,1] \text { and } t \in[0, T]
$$

and there is a strict inequality for some $(p, t)$.

Proof. See Annex 2.

Proposition 2 characterises First-order Time-Stochastic Dominance for quantile distributions and it can further easily be shown to apply to discrete data. ${ }^{8}$

${ }^{8}$ Choose an arbitrary quantile $p^{*}(t) \in[0,1]$ for any $t$ and denote $G_{1}^{-1}\left(p^{*}, t\right)=z_{2}(t)$ and $F_{1}^{-1}\left(p^{*}, t\right)=z_{1}(t)$. We need to show that $z_{1}(t) \geq z_{2}(t)$ for each $t$. Assume that $z_{1}(t)<z_{2}(t)$. By definition, $x_{2}(t)$ represents the smallest value for which equation 2 holds and for this reason $z_{1}(t)$ and $z_{2}(t)$ cannot be located on the same step of the $G_{1}^{1}(z, t)$ for any $t$. Therefore $G_{1}^{1}\left(z_{1}, t\right)<G_{1}^{1}\left(z_{2}, t\right)$. We have that $G_{1}^{1}\left(z_{1}, t\right)<G_{1}^{1}\left(z_{2}, t\right)=p^{*}(t)=F_{1}^{1}\left(z_{1}, t\right)<F_{1}^{1}\left(z_{2}, t\right)$. Thus $G_{1}^{1}\left(z_{1}, t\right)<F_{1}^{1}\left(z_{1}, t\right)$, which contradicts the initial assumption. This proves sufficiency, and necessity can be demonstrated in a very similar way. 
$F_{1}^{-1,1}(p, t)$ and $G_{1}^{-1,1}(p, t)$ are obtained by first cumulating the cashflows across time, and then reordering from lowest to highest in each time period. Taking the difference between them gives us $H_{1}^{-1,1}(p, t)$. Notice that since the quantile distribution function is just the inverse of the cumulative distribution function, $1 \mathrm{TSD}$ requires $F_{1}^{-1,1}(p, t)-G_{1}^{-1,1}(p, t) \geq 0$, i.e. the inverse of the requirement for 1TSD in terms of cumulative distributions.

In the case of Example 1, computing the quantile distributions gives us:

\begin{tabular}{|c|c|c|c|c|c|}
\hline & & Гime & period & & \\
\hline$p$ & 0 & 1 & 2 & 3 & 4 \\
\hline 0.25 & 3 & 3 & 1 & 4 & 4 \\
\hline 0.5 & 3 & 4 & 2 & 2 & 1 \\
\hline 0.75 & 4 & 3 & 2 & 3 & 0 \\
\hline 1 & 4 & 4 & 1 & 4 & 3 \\
\hline
\end{tabular}

Therefore, by Propositions 1 and $2, X>_{1 T S} Y$.

Having established First-order TSD, we can proceed from here by placing an additional restriction on the discount function and/or on the utility function. A particularly compelling case is the assumption of impatience combined with risk aversion/neutrality $-(v, u) \in V_{1} \times U_{2}$ - since few would be uncomfortable with the notion of excluding risk-seeking behaviour a priori, especially in the public sector.

Proposition 3. [First-order Time and Second-order Stochastic Dominance] $X>_{1 T 2 S} Y$ if and only if

$$
D_{1}^{2}(z, t) \geq 0, \forall z \in[a, b] \text { and } \forall t \in[0, T],
$$

and there is a strict inequality for some $(z, t)$.

Proof. See Annex 2.

It is evident from Proposition 3 and its proof that, in line with the classical approach to SD, restricting the utility function by one degree corresponds to integrating the bi-dimensional probability distribution $D_{1}^{1}(z, t)$ once more with respect to the consequence space.

Example 2. Now consider two different prospects $X$ and $Y$ :

\begin{tabular}{cc|ccccc}
\hline \multirow{2}{*}{ Prospect } & \multicolumn{5}{|c}{ Time } & period \\
\multirow{3}{*}{$X$} & Probability & 0 & 1 & 2 & 3 & 4 \\
& $1 / 4$ & -4 & -1 & 2 & 3 & 9 \\
& $1 / 4$ & -1 & -3 & 2 & 2 & 7 \\
& $1 / 4$ & -1 & -1 & 2 & 0 & 4 \\
$Y$ & $1 / 4$ & 0 & 0 & 2 & 2 & 2 \\
\hline \multirow{3}{*}{$Y$} & $1 / 4$ & -5 & -1 & 2 & 2 & 2 \\
& $1 / 4$ & -2 & -3 & -1 & 3 & 6 \\
& $1 / 4$ & -2 & 0 & 0 & 2 & 5 \\
\hline
\end{tabular}


In this example $H_{1}^{-1,1}(p, t)$ is:

\begin{tabular}{|c|c|c|c|c|c|}
\hline & & Time & period & & \\
\hline$p$ & 0 & 1 & 2 & 3 & 4 \\
\hline 0.25 & 1 & 1 & 3 & 3 & 4 \\
\hline 0.5 & 1 & 1 & 2 & 2 & 3 \\
\hline 0.75 & 1 & 0 & 2 & 0 & 2 \\
\hline 1 & 0 & 0 & 0 & 1 & -2 \\
\hline
\end{tabular}

While in the first four time periods $H_{1}^{-1,1}(p, t) \geq 0$, the opposite is true when $p=1$ in the terminal period. Therefore first-order TSD cannot be established between these two prospects. However, cumulating once more with respect to the consequence space gives $H_{1}^{-1,2}(p, t)$, which here is:

\begin{tabular}{|c|c|c|c|c|c|}
\hline & & Time & period & & \\
\hline$p$ & 0 & 1 & 2 & 3 & 4 \\
\hline 0.25 & 1 & 1 & 3 & 3 & 4 \\
\hline 0.5 & 2 & 2 & 5 & 5 & 7 \\
\hline 0.75 & 3 & 2 & 7 & 5 & 9 \\
\hline 1 & 3 & 2 & 7 & 6 & 7 \\
\hline
\end{tabular}

Thus from Proposition 3 and by extension of Proposition 2 we can say that $X>_{1 T 2 S} Y$. What this example illustrates is that, when the violation of firstorder TSD is restricted to the upper quantiles of $F_{1}^{-1,1}$ and $G_{1}^{-1,1}$, the additional restriction that $u \in U_{2}$, which excludes risk-seeking behaviour, makes it disappear, because relatively greater weight is placed on outcomes with low wealth.

The previous cases provide us with the machinery we require to offer a theorem for TSD that is generalised to the $n^{\text {th }}$ order with respect to time and the $m^{\text {th }}$ order with respect to risk.

Proposition 4. $\left[n^{\text {th }}\right.$-order Time and $m^{\text {th }}$-order Stochastic Dominance] $X n^{\text {th }}$-order time and $m^{\text {th }}$-order stochastic dominates $Y$ if

$$
\begin{gathered}
i) D_{i+1}^{j+1}(b, T) \geq 0, \\
i i) D_{n}^{j+1}(b, t) \geq 0, \forall t \in[0, T], \\
i i i) D_{i+1}^{m}(z, T) \geq 0, \forall z \in[a, b], \\
i v) D_{n}^{m}(z, t) \geq 0, \forall z \in[a, b] \text { and } \forall t \in[0, T],
\end{gathered}
$$

with (iv) holding as a strong inequality over some sub interval and where $i=$ $\{0, \ldots, n-1\}$ and $j=\{0, \ldots, m-1\}$.

The proof is constructed as a simple extension of the previous analysis. Integrating by parts repeatedly, we obtain: 


$$
\begin{aligned}
N P V_{E_{F}, v}-N P V_{E_{G}, v}= & \sum_{i=1}^{n-1}(-1)^{j+1} u^{j}(b)\left[\sum_{j=0}^{m-1}(-1)^{j} v^{j}(T) D_{i+1}^{j+1}(b, T)\right] \\
& +\sum_{i=1}^{n-1}(-1)^{j+1}(-1)^{n} u^{j}(b) \int_{0}^{T} v^{n}(t) D_{n}^{j+1}(b, t) d t+ \\
& +\sum_{j=0}^{m-1}(-1)^{i}(-1)^{m-1} v^{i}(T) \int_{a}^{b} u^{m}(z) D_{i+1}^{m}(z, T) d z+ \\
& +(-1)^{m+n+1} \int_{a}^{b} \int_{0}^{T} v^{n}(t) u^{m}(z) D_{n}^{m}(z, t) d t d z .
\end{aligned}
$$

From which the statement of the Proposition follows immediately.

\section{Almost Time-Stochastic Dominance}

In practice, the usefulness of what we might call 'exact' dominance analysis can be limited, since even a very small violation of the conditions for dominance is sufficient to render the rules unable to order investments. As the example in the Introduction showed, if a violation exists in particular at the lower bound of the domain of the cumulative distribution functions, then no amount of restrictions will make it vanish. Put another way, the downside of a flexible, non-parametric approach is that the broad classes of preference on which the dominance criteria are based include a small subset of 'extreme' or 'pathological' functions, whose implications for choice would be regarded by many as perverse. ${ }^{9}$ Leshno and Levy (2002) recognised this problem in the context of SD and developed a theory of Almost Stochastic Dominance (Almost SD), according to which restrictions are placed on the derivatives of the utility function, so that extreme preferences are excluded (see Annex 1). ${ }^{10}$ Dominance relations between risky prospects are then characterised for 'almost' all decision-makers.

It is obvious that exact TSD faces the same practical constraints as exact SD. In this section we therefore extend our theory to 'Almost TSD', excluding extreme combinations of time and risk preferences so that prospects can still be ranked. In particular, by extending the theory to our bi-dimensional time-risk

\footnotetext{
${ }^{9}$ What is 'extreme' is clearly subjective, an obvious difficulty faced by the Almost SD approach. However, Levy et al. (2010) offer an illustration of how to define it using laboratory data on participant choices when faced with binary lotteries. Extreme risk preferences are marked out by establishing gambles that all participants are prepared to take. By making the conservative assumption that no participant has extreme risk preferences, the most riskseeking and risk-averse participants mark out the limits, and preferences outside these limits can be considered extreme.

${ }^{10}$ Tzeng et al. (2012) showed that Leshno and Levy's theorem for Almost Second-order Stochastic Dominance is incorrect and re-defined the concept. They also extended the results to higher orders.
} 
set-up, we define and characterise Almost First-order TSD and Almost Firstorder Time and Second-order Stochastic Dominance. In doing so, the attention of the analysis shifts subtly to asking; how many preference combinations must be excluded in order to obtain a ranking? Put another way, how much smaller is the space for agreement? In general, the less that need be excluded, the better.

Let us start with Almost First-order TSD. Our basic approach is analogous to Leshno and Levy (2002) in that we measure the violation of 1TSD relative to the non-violation of 1TSD, and give the resulting, relative measure of violation meaning by linking it with a restriction on time and risk preferences. To see this, begin by defining the set of realisations $z \in[a, b]$ where there is a violation of First-order TSD as $S_{1}^{1}$ :

$$
S_{1}^{1}\left(D_{1}^{1}\right)=\left\{z \in[a, b], t \in[0, T]: D_{1}^{1}(z, t)<0\right\} .
$$

We also explicitly define $S^{1, T}$ as the subset of $S_{1}^{1}$ when $t=T$, i.e. the difference between the single-dimensional cumulative distributions over the consequence space at time $T$ :

$$
S^{1, T}\left(D_{1}^{1}\right)=\left\{z \in[a, b], t=T: D_{1}^{1}(z)<0\right\} .
$$

Definition 2. [Almost First-order Time-Stochastic Dominance] X dominates $Y$ by Almost First-order Time-Stochastic Dominance, denoted $X>_{A 1 T S}$ $Y$, if and only if

$$
\begin{aligned}
& \text { i) } \iint_{S_{1}^{1}}-D_{1}^{1}(z, t) d z d t \leq \gamma_{1} \int_{0}^{T} \int_{a}^{b}\left|D_{1}^{1}(z, t)\right| d z d t \text { and } \\
& \text { ii) } \int_{S^{1, T}}-D_{1}^{1}(z, T) d z \leq \varepsilon_{1 T} \int_{a}^{b}\left|D_{1}^{1}(z, T)\right| d z .
\end{aligned}
$$

Proposition 5. [A1TSD] $X>_{A 1 T S} Y$ if and only if, for all $(v, u) \in\left(V_{1} \times\right.$ $\left.U_{1}\right)\left(\gamma_{1}\right)$ and $u \in U_{1}\left(\varepsilon_{1 T}\right)$,

$$
N P V_{v, u}(X) \geq N P V_{v, u}(Y)
$$

Proof. See Annex 2.

The definition of Almost First-order TSD contains two measures of the violation of exact First-order TSD. $\gamma_{1}$ measures the cumulative violation of the non-negativity condition on $D_{1}^{1}$ over all $t$, relative to the total volume enclosed between the distributions over all $t$, while $\varepsilon_{1 T}$ measures the violation of the same condition at time $T$ only, relative to the total area enclosed between the distributions at that time. For the sake of an easy exposition, let us explain the latter violation measure first, $\varepsilon_{1 T}$. This has, in fact, the same interpretation in terms of utility as the corresponding violation measure in Leshno and Levy (2002). The difference is that in our bi-dimensional framework it is for $t=T$. Adapting their theorem to our context, for every $0<\varepsilon_{1 T}<0.5$, define the following subset of $U_{1}$ :

$$
U_{1}\left(\varepsilon_{1 T}\right)=\left\{u \in U_{1}: \frac{u^{\prime}(z)}{\inf \left[u^{\prime}(z)\right]} \leq\left[\frac{1}{\varepsilon_{1 T}}-1\right], \forall z \in[a, b], t=T\right\} .
$$


$U_{1}\left(\varepsilon_{1 T}\right)$ is the set of non-decreasing utility functions with the added restriction that the ratio between maximum and minimum marginal utility is bounded by $\frac{1}{\varepsilon_{1 T}}-1$, i.e. extreme concavity/convexity is ruled out. It is easiest to see what this restriction entails in the case of $u \in U_{1}\left(\varepsilon_{1 T}\right)$, where $u^{\prime \prime}(z)$ is monotonic. Then we are restricting how much (little) marginal utility members of the class of functions associate with low wealth levels at the same time as restricting how little (much) marginal utility they associate with high wealth levels. Further narrowing the scope to the very common case of utility functions with constant elasticity of marginal utility, the restriction is on the absolute value of the elasticity $-\left|\frac{u^{\prime \prime}(z) z}{u^{\prime}(z)}\right|-$ such that it cannot be large negative or large positive, and the larger is $\varepsilon_{1 T}$ the smaller $\left|\frac{u^{\prime \prime}(z) z}{u^{\prime}(z)}\right|$ must be. Of course this is merely an illustration - the set of utility functions $U_{1}\left(\varepsilon_{1 T}\right)$ is much larger than the constant-relative-risk aversion functions alone. In the limit as $\varepsilon_{1 T}$ approaches 0.5 , the only function in $U_{1}\left(\varepsilon_{1 T}\right)$ is linear utility, where $u^{\prime \prime}(z)=0$. Conversely as $\varepsilon_{1 T}$ approaches zero, $U_{1}\left(\varepsilon_{1 T}\right)$ coincides with $U_{1}$.

$\gamma_{1}$ is defined in terms of the product of the marginals of the discount and utility functions as follows:

$$
\begin{gathered}
\left(V_{1} \times U_{1}\right)\left(\gamma_{1}\right)=\left\{v \in V_{1}, u \in U_{1}: \frac{-v^{\prime}(t) u^{\prime}(z)}{\inf \left[-v^{\prime}(t) u^{\prime}(z)\right]} \leq\left[\frac{1}{\gamma_{1}}-1\right],\right. \\
\forall z \in[a, b], \forall t \in[0, T]\}
\end{gathered}
$$

$\left(V_{1} \times U_{1}\right)\left(\gamma_{1}\right)$ is the set of all combinations of decreasing pure time discount function and non-decreasing utility function, with the added restriction that the ratio between the maximum and minimum products of $-v^{\prime}(t) u^{\prime}(z)$ is bounded by $\frac{1}{\gamma_{1}}-1$. The supremum (infimum) of $-v^{\prime}(t) u^{\prime}(z)$ is attained when $v^{\prime}(t)<0$ is the infimum (supremum) of its set and $u^{\prime}(z) \geq 0$ is the supremum (infimum) of its. Therefore the combinations of preferences that we are excluding here will tend to comprise extreme concavity or convexity of the utility and discount functions somewhere on their respective domains. All decision makers exhibiting the non-extreme combination of preferences expressed by the discount and utility functions $(v, u) \in\left(V_{1} \times U_{1}\right)\left(\gamma_{1}\right)$ and $u \in U_{1}\left(\varepsilon_{1 T}\right)$ will prefer $X$ to $Y$ if and only if the condition in Proposition 5 is satisfied.

Moving now to Almost First-order Time and Second-order Stochastic Dominance, parcel out the subset of realisations $S_{1}^{2}$ where $D_{1}^{2}<0$, i.e. where the condition for exact First-order Time and Second-Order Stochastic Dominance is violated:

$$
S_{1}^{2}\left(D_{1}^{2}\right)=\left\{z \in[a, b], t \in[0, T]: D_{1}^{2}(z, t)<0\right\} .
$$

Further explicitly define $S^{2, T}$ as the subset of $S_{1}^{2}$ when $t=T$ :

$$
S^{2, T}\left(D_{1}^{2}\right)=\left\{z \in[a, b], t=T: D_{1}^{2}(z)<0\right\} .
$$

And in this case we also need to define a subset of realisations where $D_{1}^{2}(b, t)<0$, for any $t$ where $z=b$ :

$$
S_{1, b}\left(D_{1}^{2}\right)=\left\{z=b, t \in[0, T]: D_{1}^{2}(t)<0\right\} .
$$


Definition 3. [Almost First-order Time and Second-order Stochastic Dominance] $X$ Almost First-order Time and Second-order Stochastic Dominates $Y$, denoted $X>_{A 1 T 2 S} Y$ if and only if

$$
\begin{gathered}
\text { i) } \iint_{S_{1}^{2}}-D_{1}^{2}(z, t) d z d t \leq \gamma_{1,2} \int_{0}^{T} \int_{a}^{b}\left|D_{1}^{2}(z, t)\right| d z d t, \\
\text { ii) } \int_{S^{2, T}}-D_{1}^{2}(z, T) d z \leq \varepsilon_{2 T} \int_{a}^{b}\left|D_{1}^{2}(z, T)\right| d z, \\
\text { iii) } \int_{S_{1, b}} D_{1}^{2}(b, t) d t \leq \lambda_{1 b} \int_{0}^{T}\left|D_{1}^{2}(b, t)\right| d t,: \text { and } \\
\text { iv) } D_{1}^{2}(b, T) \geq 0
\end{gathered}
$$

Proposition 6. [A 1T2SD] $X>_{A 1 T 2 S} Y$ if and only if, for all $(v, u) \in\left(V_{1} \times\right.$ $\left.U_{2}\right)\left(\gamma_{1,2}\right), u \in U_{2}\left(\varepsilon_{2 T}\right)$ and $v \in V_{1}\left(\lambda_{1 b}\right)$,

$$
N P V_{v, u}(X) \geq N P V_{v, u}(Y) .
$$

Proof. See Annex 2.

Notice that the definition of Almost First-order Time and Second-order Stochastic Dominance has a similar structure to Proposition 4. It contains three measures of the violation of strict dominance, as well as the requirement that $D_{1}^{2}(b, T) \geq 0$, i.e. that the difference between the undiscounted mean values of projects $X$ and $Y$ respectively is at least zero. First, $\gamma_{1,2}$ measures the relative violation of the non-negativity condition on $D_{1}^{2}$ over all $t$. It is equivalent to the following restriction on combined time and risk preferences:

$$
\begin{gathered}
\left(V_{1} \times U_{2}\right)\left(\gamma_{1,2}\right)=\left\{v \in V_{1}, u \in U_{2}: \frac{v^{\prime}(t) u^{\prime \prime}(z)}{\inf \left[v^{\prime}(t) u^{\prime \prime}(z)\right]} \leq\left[\frac{1}{\gamma_{1,2}}-1\right] .\right. \\
\forall z \in[a, b], \forall t \in[0, T]\}
\end{gathered}
$$

The set $\left(V_{1} \times U_{2}\right)\left(\gamma_{1,2}\right)$ represents all combinations of decreasing pure time discount functions and non-decreasing, weakly concave utility functions, with the added restriction that the ratio between the maximum and minimum of $v^{\prime}(t) u^{\prime \prime}(z)$ is bounded by $\frac{1}{\gamma_{1,2}}-1$. The supremum (infimum) of $v^{\prime}(t) u^{\prime \prime}(z)$ is attained when $v^{\prime}(t)<0$ and $u^{\prime \prime}(z) \leq 0$ are the suprema (infima) of their respective sets, and these sets are defined with respect to all realisations and time-periods.

Second, $\varepsilon_{2 T}$ measures the relative violation of the non-negativity condition on $D_{1}^{2}$ at time $T$ only. As per Leshno and Levy (2002), for every $0<\varepsilon_{2 T}<0.5$,

$$
U_{2}\left(\varepsilon_{2 T}\right)=\left\{u \in U_{2}: \frac{-u^{\prime \prime}(z)}{\inf \left[-u^{\prime \prime}(z)\right]} \leq\left[\frac{1}{\varepsilon_{2 T}}-1\right], \forall z \in[a, b], t=T\right\} .
$$

$U_{2}\left(\varepsilon_{2 T}\right)$ is the set of non-decreasing, weakly concave utility functions with the added restriction that the ratio between maximum and minimum $u^{\prime \prime}(z)$ is bounded by $\frac{1}{\varepsilon_{2 T}}-1$. Therefore large changes in $u^{\prime \prime \prime}(z)$ with respect to $z$ are excluded, where only realisations at time $T$ are considered.

Third, we need to measure a violation of the non-negativity condition on the integral with respect to time of $D_{1}^{2}(b, t)$. We denote this $\lambda_{1 b}$ and it is equivalent to restricting time preferences as follows:

$$
V_{1}\left(\lambda_{1 b}\right)=\left\{v \in V_{1}: \frac{-v^{\prime}(t)}{\inf \left[-v^{\prime}(t)\right]} \leq v\left[\frac{1}{\lambda_{1 b}}-1\right], z=b, \forall t \in[0, T]\right\} .
$$


$V_{1}\left(\lambda_{1 b}\right)$ is the set of decreasing pure time discount functions with the added restriction that the ratio between maximum and minimum $v^{\prime}(t)$ is bounded by $\frac{1}{\lambda_{1 b}}-1$. Hence large changes in $v^{\prime \prime}(t)$ are excluded.

Example 4. Consider the following two prospects:

\begin{tabular}{cc|ccccc}
\hline \multirow{3}{*}{ Prospect } & Probability & 0 & Time & period & & \\
\hline \multirow{3}{*}{$X$} & $1 / 4$ & -5 & -3 & 0 & 4 & 7 \\
& $1 / 4$ & 0 & -3 & 1 & 2 & 10 \\
& $1 / 4$ & 0 & -2 & 1 & 3 & 9 \\
& $1 / 4$ & 0 & 0 & 0 & 1 & 1 \\
\hline \multirow{3}{*}{$Y$} & $1 / 4$ & -5 & -1 & 0 & 3 & 9 \\
& $1 / 4$ & -4 & -2 & -1 & 0 & 1 \\
& $1 / 4$ & -2 & -3 & 1 & 1 & 5 \\
& $1 / 4$ & -2 & -1 & -1 & 2 & 1 \\
\hline
\end{tabular}

In this example $H_{1}^{-1,1}$ is:

\begin{tabular}{|c|c|c|c|c|c|}
\hline & & Time & period & & \\
\hline$p$ & 0 & 1 & 2 & 3 & 4 \\
\hline 0.25 & 0 & -2 & -1 & 3 & 8 \\
\hline 0.5 & 4 & 3 & 4 & 3 & 4 \\
\hline 0.75 & 2 & 3 & 3 & 4 & 8 \\
\hline 1 & 2 & 3 & 4 & 4 & 5 \\
\hline
\end{tabular}

First-order TSD cannot be established between these two prospects. Moreover it can easily be shown that the occurrence of the violation in the lowest quantile of $H_{1}^{-1,1}$, in early time periods, means that the violation will persist despite infinitely repeated cumulation with respect to time and/or the consequence space. However, it is quite evident from the tables that $X$ performs better than $Y$ most of the time, so let us inspect this example within the framework of Almost TSD. Doing the necessary calculations:

\begin{tabular}{cc|ccc}
\hline A1TSD & \multicolumn{3}{|c}{ A1T2SD } \\
$\gamma_{1}$ & $\varepsilon_{1 T}$ & $\gamma_{2}$ & $\varepsilon_{2 T}$ & $\gamma_{1 b}$ \\
\hline 0.04 & 0 & 0.02 & 0 & 0 \\
\hline
\end{tabular}

The small violations reflect what is intuitively obvious from $H_{1}^{-1,1}(p, t)$, namely that only a small restriction on the combination of classes of discount and utility functions is required in order for dominance to be established, since $F<G$ most of the time in most quantiles. 


\section{$5 \quad$ Modelling climate mitigation policies}

We now turn to our application of the theory of TSD and Almost TSD to climate-change mitigation. The question we ask is; can we make choices on emissions abatement, without having to agree on how precisely to structure and parameterise time and risk preferences in economic models of climate mitigation? Are there combinations of whole classes of discount and utility functions, for which it is possible to say that some abatement policies are preferred to others?

To offer answers to these questions, we generate quantile data on the consumption benefits of emissions reduction policies using the DICE model. DICE essentially couples a Ramsey-Cass-Koopmans growth model to a simple climate model by generating carbon dioxide emissions as a side-effect of production, and by connecting climate change back to output and welfare via a so-called damage function. The model is described fully in Nordhaus (2008) and so we confine our discussion here to changes that we have made.

\section{A stochastic version of DICE}

Standard versions of DICE are deterministic, with fixed parameters. This is a poor fit to the problem of evaluating climate policy, however, because risk is a central element. Therefore we use a stochastic version of DICE, developed by Dietz and Asheim (2012). This version randomises eight parameters in the model so that Monte Carlo simulation can be undertaken. Table 1 lists the eight parameters, and the form and parameterisation of the probability density functions assigned to them.

The eight random parameters were originally selected by Nordhaus (2008), based on his broader assessment of which of all the model's parameters had the largest impact on the value of policies. The first four parameters in Table 1 play a role in determining $\mathrm{CO}_{2}$ emissions. In one-sector growth models like DICE, $\mathrm{CO}_{2}$ emissions are directly proportional to output, which is in turn determined in significant measure by productivity (i) and the stock of labour (ii). However, while $\mathrm{CO}_{2}$ emissions are proportional to output, the proportion is usually assumed to decrease over time due to autonomous structural and technical change (iii). A further check on industrial $\mathrm{CO}_{2}$ emissions is provided in the long run by the finite total remaining stock of fossil fuels (iv).

The fifth uncertain parameter is the price of a $\mathrm{CO}_{2}$-abatement backstop technology. In DICE, the coefficient of the abatement cost function depends on the backstop price, hence we obtain abatement cost uncertainty as a result of backstop price uncertainty.

The sixth and seventh parameters in Table 1 capture important uncertainties in climate science. Parameter (vi) captures uncertainty about the carbon cycle, via the proportion of $\mathrm{CO}_{2}$ in the atmosphere in a particular time-period, which dissolves into the upper ocean in the next period. Uncertainty about the relationship between a given stock of atmospheric $\mathrm{CO}_{2}$ and temperature is captured by specifying a random climate-sensitivity parameter (vii). The climate sensi- 
Table 1: Uncertain parameters for simulation of DICE.

\begin{tabular}{lcccc}
\hline Parameter & Units & $\begin{array}{c}\text { Functional } \\
\text { form }\end{array}$ & Mean & $\begin{array}{c}\text { Standard } \\
\text { deviation }\end{array}$ \\
\hline \hline $\begin{array}{l}\text { (i) Initial growth } \\
\text { rate of TFP }\end{array}$ & $\begin{array}{c}\text { Per } \\
\text { year }\end{array}$ & Normal & 0.0092 & 0.004 \\
\hline $\begin{array}{l}\text { (ii) Asymptotic } \\
\text { global population }\end{array}$ & Millions & Normal & 8600 & 1892 \\
\hline $\begin{array}{l}\text { (iii) Rate of } \\
\text { decarbonisation }\end{array}$ & $\begin{array}{l}\text { Per } \\
\text { year }\end{array}$ & Normal & -0.007 & 0.002 \\
\hline $\begin{array}{l}\text { (iv) Total resources } \\
\text { of fossil fuels }\end{array}$ & $\begin{array}{c}\text { Billion tons } \\
\text { of carbon }\end{array}$ & Normal & 6000 & 1200 \\
\hline $\begin{array}{l}\text { (v) Price of back- } \\
\text { stop technology }\end{array}$ & $\begin{array}{c}\text { US\$ per ton of } \\
\text { carbon replaced }\end{array}$ & Normal & 1170 & 468 \\
\hline $\begin{array}{l}\text { (vi) Transfer coefficient } \\
\text { in carbon cycle }\end{array}$ & $\begin{array}{c}\text { Per } \\
\text { decade }\end{array}$ & Normal & 0.189 & 0.017 \\
\hline $\begin{array}{l}\text { (vii) Climate } \\
\text { sensitivity }\end{array}$ & $\begin{array}{c}{ }^{\circ} \text { C per doubling of } \\
\text { atmospheric CO }\end{array}$ & Log- & $1.099^{*}$ & $0.3912^{*}$ \\
\hline $\begin{array}{l}\text { (viii) Damage function } \\
\text { coefficient } \alpha_{3}\end{array}$ & $\begin{array}{c}\text { Fraction of } \\
\text { global output }\end{array}$ & Normal & 0.082 & 0.028 \\
\hline \hline
\end{tabular}

*In natural logarithm space.

tivity is the increase in global mean temperature, in equilibrium, that results from a doubling of the atmospheric stock of $\mathrm{CO}_{2}$. In simple climate models like DICE's, it is critical in determining how fast and how far the planet is forecast to warm in response to emissions. There is by now much evidence, derived from a variety of approaches (see Meehl et al., 2007, and Roe and Baker, 2007), that the probability density function for the climate sensitivity has a positive skew.

The eighth and final uncertain parameter is one element of the damage function linking temperature and utility-equivalent losses in output. In Dietz and Asheim's (2012) version of DICE, the damage function has the following form:

$$
\Omega(t)=\frac{1}{1+\alpha_{1} \Upsilon(t)+\alpha_{2} \Upsilon(t)^{2}+\left[\tilde{\alpha}_{3} \Upsilon(t)\right]^{7}},
$$

where $\Omega$ is the proportion of output lost, $\Upsilon$ is the increase in global mean temperature over the pre-industrial level, and $\alpha_{i}, i \in\{1,2,3\}$ are coefficients. $\tilde{\alpha}_{3}$ is a normally distributed random coefficient (viii), so the higher-order term $\left[\tilde{\alpha}_{3} \Upsilon(t)\right]^{7}$ captures the uncertain prospect that significant warming of the planet could be accompanied by a very steep increase in damages. That such a possibility exists has been the subject of recent controversy, with the approaches of Nordhaus (2008) and Weitzman (2012) marking out opposing stances. The controversy exists, because there is essentially no empirical evidence to support calibration of the damage function at high temperatures (Dietz, 2011; Tol, 2012); instead there are simply beliefs. In standard DICE, $\alpha_{3}=0$, thus there 
is no higher-order effect and $5^{\circ} \mathrm{C}$ warming, as a benchmark for a large temperature increase, results in a loss of $6 \%$ of output. By contrast Weitzman (2012) suggests a functional form which can be approximated by $\alpha_{3}=0.166$. Here $\tilde{\alpha}_{3}$ is calibrated such that the Nordhaus and Weitzman positions represent minus/plus three standard deviations respectively, and at the mean $5^{\circ} \mathrm{C}$ warming results in a loss of utility equivalent to around $7 \%$ of output.

Random parameters (i)-(viii), alongside the model's remaining non-random parameters and initial conditions (as per Nordhaus, 2008), are inputs to a Monte Carlo simulation. In particular, a Latin Hypercube sample of 1000 runs of the model is taken. Each run solves the model for a particular policy, which as described below is a schedule of values for the rate of control of $\mathrm{CO}_{2}$ emissions. From this is produced a schedule of distributions of consumption per capita (where consumption per capita is equivalent to a cashflow in our theory), which is the focus of the Time-Stochastic Dominance analysis.

\section{Policies to be evaluated}

We evaluate a set of five exogenous policies governing the rate of control of $\mathrm{CO}_{2}$ emissions, plus a sixth path representing a forecast of emissions in the absence of policy-driven controls, i.e. 'business as usual' or BAU. Our aims in generating this set are to achieve consistency with the modelling framework described just now, as well as a degree of representativeness of the broader policy literature on emissions reduction trajectories (e.g. Clarke et al., 2014).

Each of the five policies limits the atmospheric stock of $\mathrm{CO}_{2}$ to a pre-specified level. This approach is very similar to many real policy discussions, which aim for a 'stabilisation' level of atmospheric $\mathrm{CO}_{2}$ in the very long run. In order to render the policies consistent with the assumptions we make, we use the stochastic version of DICE itself to generate the five policy paths. BAU is the baseline scenario from Nordhaus (2008).

The control variable is the percentage reduction in industrial $\mathrm{CO}_{2}$ emissions relative to uncontrolled emissions (i.e. not relative to BAU). Each policy path is generated by solving a stochastic optimisation problem, whereby the schedule of emissions cuts is chosen to minimise abatement $\operatorname{costs}^{11}$ subject to the constraint that the mean atmospheric stock of $\mathrm{CO}_{2}, M^{A T}(t) \leq \overline{M^{A T}}$, where $\overline{M^{A T}} \in$ $\{450,500,550,600,650\}$ and where the units are parts per million volume. This is done under intial uncertainty about parameters (i)-(vi), since these affect the cost of abatement and its impact on atmospheric $\mathrm{CO}_{2}$. As with most of the literature, we assume that the cost-effective path at $t=0$ is adhered to, despite the resolution of all uncertainty just after $t=0$, which should be contrasted with more complex approaches that model learning and consequent revisions to the controls (e.g. Kelly and Kolstad, 1999; Lemoine and Traeger, 2014).

\footnotetext{
${ }^{11}$ Of course, what is cost-effective depends on the social objective, so for this part of the analysis we cannot avoid pre-specifying and parameterising the social welfare and utility functions. For this purpose, we make representative choices, namely that $\delta(t)=1.5 \%, \forall t$, and the coefficient of relative risk aversion is two.
} 
In an integrated assessment model such as DICE, and especially in running Monte Carlo simulation, solving this cost-minimisation problem is a non-trivial computational challenge. We solve it using a genetic algorithm (Riskoptimizer) and with two modifications to the basic optimisation problem. ${ }^{12}$ In addition, we limit the Latin Hypercube Sample to 250 draws just for this task. ${ }^{13}$

Figure 1: Abatement policies in terms of the emissions control rate.

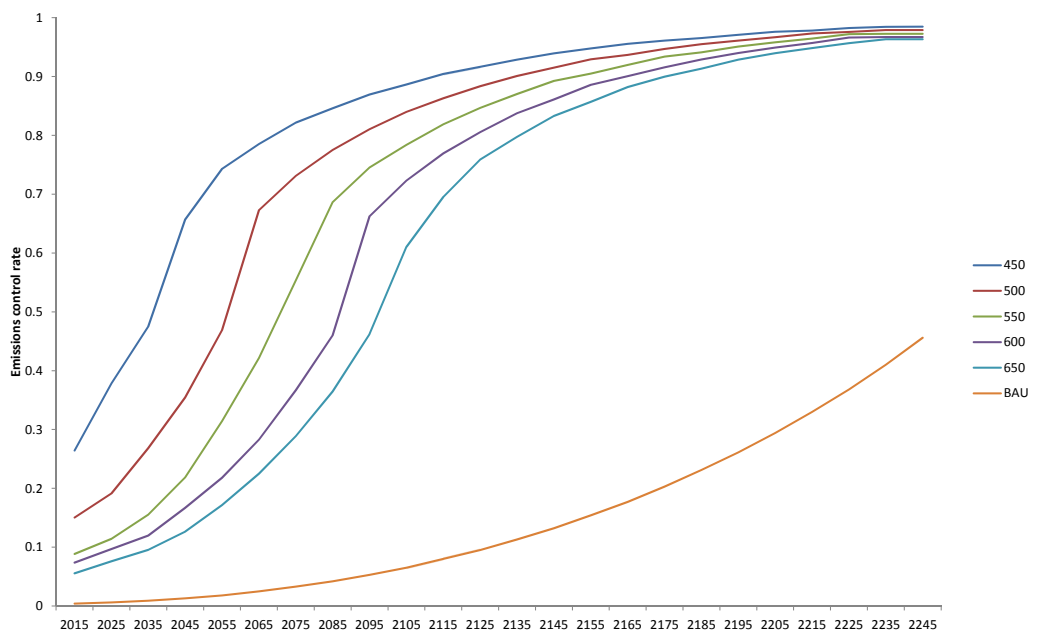

\footnotetext{
${ }^{12}$ First, we only solve for the emissions control rate from 2015 to 2245 inclusive, rather than all the way out to 2395 . This considerably reduces the scope of the optimisation problem, in return for making little difference to the results, since, in the standard version of DICE, the optimal emissions control rate is $100 \%$ when $t>2245$, as the backstop abatement technology becomes the lowest cost energy technology. Our first period of emissions control is 2015, since 2005, the first period of the model, is in the past. Second, we guide the optimisation by imposing the soft constraint that the emissions control rate is non-decreasing everywhere (via an exponential penalty function when the control rate decreases between any two timeperiods). We were able to verify that the algorithm's best solution satisfied the property of non-decreasingness in the emissions control rate, and that no solution was found which returned lower costs, where the control rate was decreasing at any point.

${ }^{13}$ In order to ensure comparability with the results of the Time-Stochastic Dominance analysis, the smaller sample is calibrated on the sample statistics of the larger sample.
} 


\section{Results}

\section{Time-Stochastic Dominance analysis}

We carry out the TSD analysis in two parts. In the first part we examine whether any of the abatement policies Time-Stochastic Dominates BAU. That is to ask, can we use the analysis to establish that there is a space for agreement on acting to reduce greenhouse gas emissions by some non-trivial amount? This would already be of considerable help in understanding the scope of the debate about climate mitigation. In the second part we use the framework to compare the emissions reduction policies themselves - can we further use the framework to discriminate between the set of policies, so that we end up with a relatively clear idea of the policy that would be preferred?

Recall from Propositions 1 and 2 that First-order TSD requires $H_{1}^{-1,1}(p, t) \geq$ $0, \forall z, t$, with at least one strict inequality. Figure 2 plots $H_{1}^{-1,1}(p, t)$ when $\overline{M^{A T}} \in\{450,500,550,600,650\}$ is compared with BAU. With the red shaded areas indicating a violation of the non-negativity condition on $H_{1}^{-1,1}(p, t)$, visual inspection is sufficient to establish that no abatement policy First-order Time-Stochastic Dominates BAU, not even the most accommodating 650ppm concentration limit.

Although First-order TSD cannot be established between abatement and BAU, it could still be that one or more of the policies is preferred to BAU according to First-order Time and Second-order Stochastic Dominance. Proposition 3 and its quantile equivalent show that this requires $H_{1}^{-1,2}(p, t) \geq 0, \forall z, t$, with at least one strict inequality. Figure 3 plots $H_{1}^{-1,2}$ when each abatement policy is compared with BAU. Again, it is straightforward to see that the condition for exact First-order Time and Second-order Stochastic Dominance is not satisfied for any of the policies. This is because, for all policies, there exists a time-period in which the lowest level of consumption per capita is realised under the mitigation policy rather than BAU.

Unable to establish exact TSD of abatement over BAU, we now turn to analysing Almost TSD. In particular, we look at both Almost First-order TSD as set out in Definition 2 and Proposition 5, and Almost First-order Time and Second-order Stochastic Dominance as set out in Definition 3 and Proposition 6 . Recall that $\gamma_{k}$ denotes the overall volume of violation of exact TSD relative to the total volume enclosed between $G_{i}^{j}$ and $F_{i}^{j} \cdot \varepsilon_{k T}$ is the violation of exact TSD in the final time-period only, while $\lambda_{1 b}$ is the violation of exact First-order Time and Second-order Stochastic Dominance with respect to realisation $b$. As $\gamma_{k}, \varepsilon_{k T}, \lambda_{1 b} \rightarrow 0.5$, the volume/area of violation accounts for half of the entire volume/area between the cumulative distributions being compared, while as $\gamma_{k}, \varepsilon_{k T}, \lambda_{1 b} \rightarrow 0$ there is no violation.

What is striking about the results of analysing Almost TSD in Table 2 is how small the violations are. For all of the policies, in particular it is the violation of exact First-order TSD that is tiny relative to the total volume/area between the distributions. Therefore we have a formal result showing that everyone would prefer any of the abatement policies to BAU, as long as their time and 
Figure 2: $H_{1}^{-1,1}(p, t)$ for $\overline{M^{A T}} \in\{450,500,550,600,650\}$.

$650 \mathrm{ppm}$

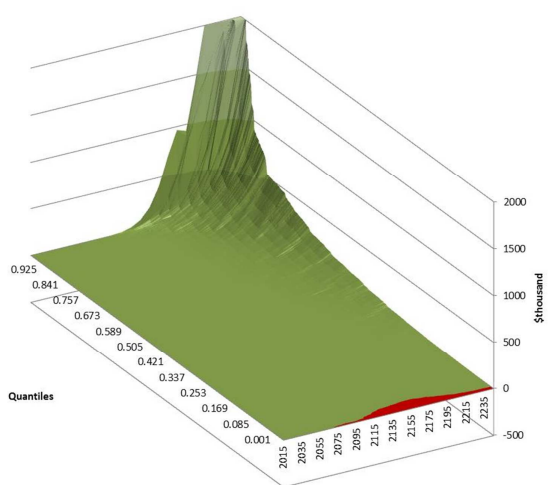

$550 \mathrm{ppm}$

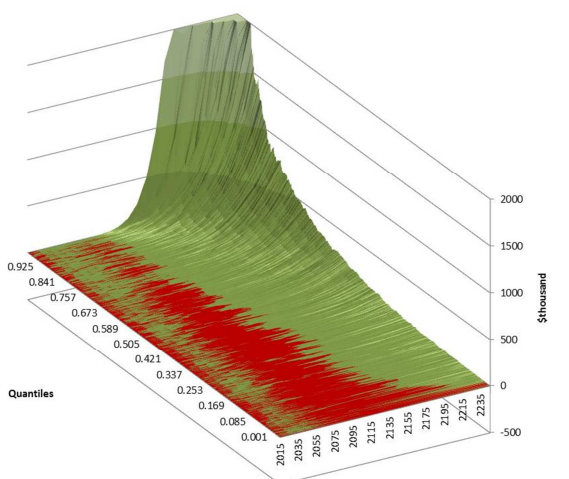

450ppm

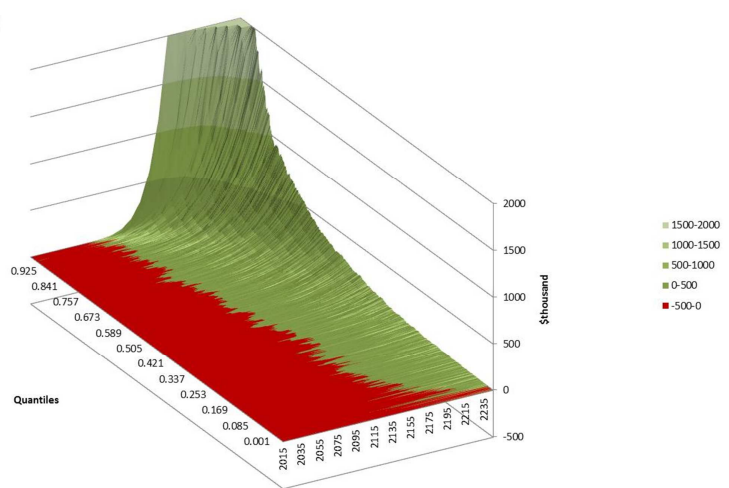

$600 \mathrm{ppm}$

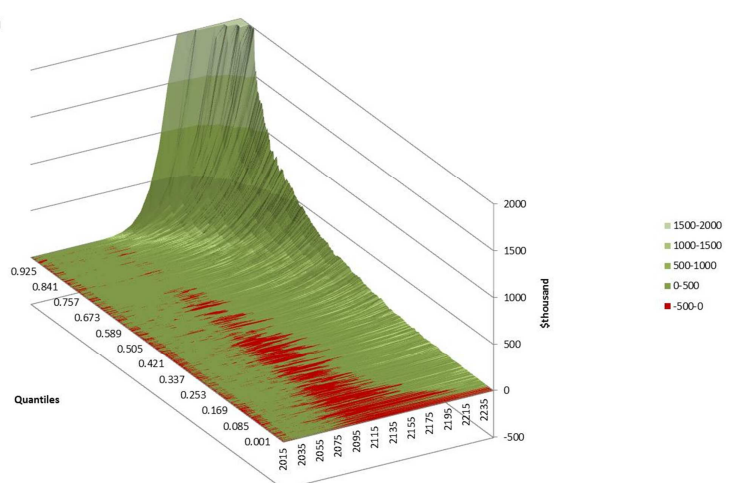

500ppm

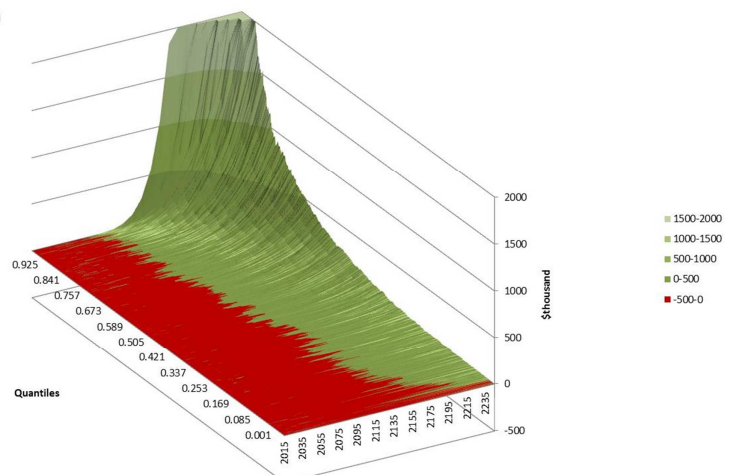


Figure 3: $H_{1}^{-1,2}(p, t)$ for $\overline{M^{A T}} \in\{450,500,550,600,650\}$.

650ppm

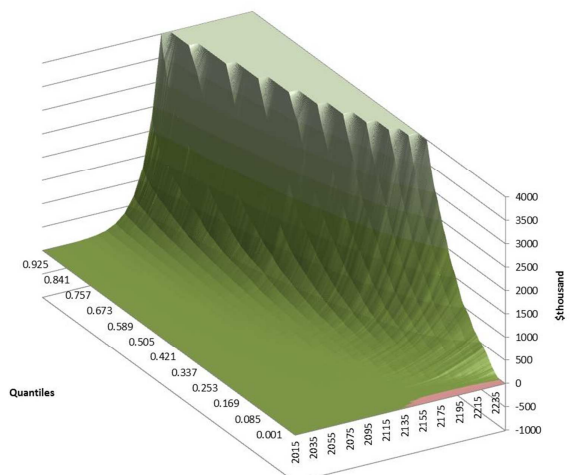

$550 \mathrm{ppm}$

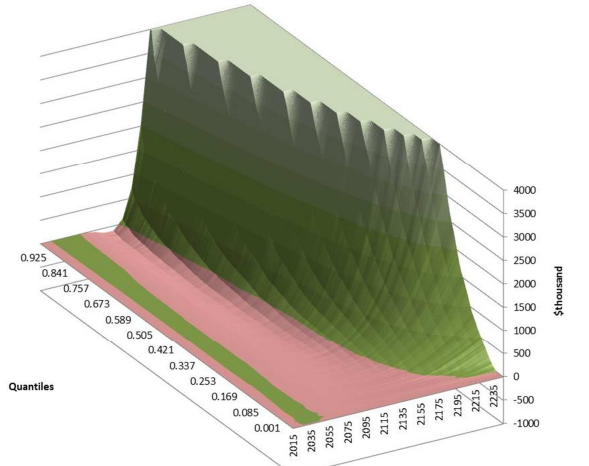

450ppm



600ppm

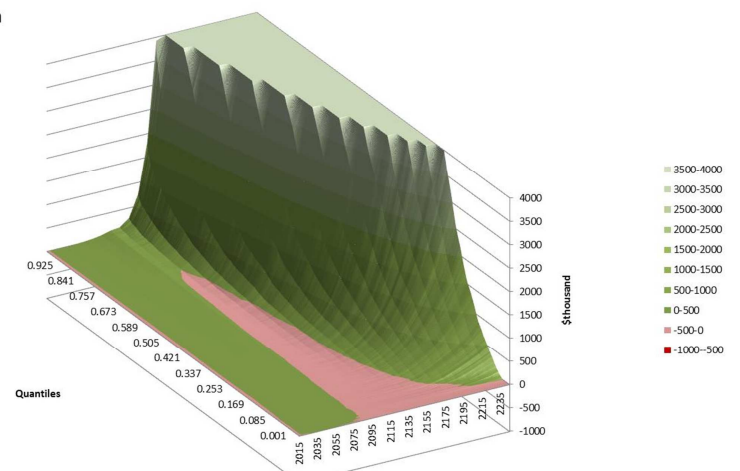

500ppm

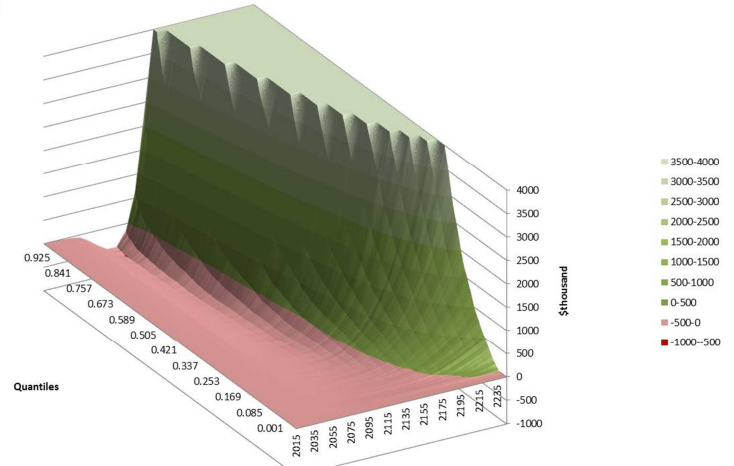


Table 2: Violations of exact First-order TSD and exact First-order Time and Second-order Stochastic Dominance.

\begin{tabular}{c|cc|ccc}
\hline $\mathrm{CO}_{2}$ limit $(\mathrm{ppm})$ & $\gamma_{1}$ & $\varepsilon_{1 T}$ & $\gamma_{1,2}$ & $\varepsilon_{2 T}$ & $\lambda_{1 b}$ \\
\hline \hline 650 & 0.00009 & 0.00003 & 0.00002 & $8 \mathrm{E}-07$ & 0 \\
600 & 0.00045 & 0.00003 & 0.00045 & $2 \mathrm{E}-06$ & $6.01 \mathrm{E}-08$ \\
550 & 0.00092 & 0.00003 & 0.00231 & $2 \mathrm{E}-06$ & 0.00014 \\
500 & 0.00188 & 0.00004 & 0.00605 & $3 \mathrm{E}-06$ & 0.00086 \\
450 & 0.00388 & 0.00004 & 0.01363 & $4 \mathrm{E}-06$ & 0.00245 \\
\hline
\end{tabular}

Table 3: First-order TSD analysis of abatement policies against each other.

\begin{tabular}{c|cc|cc|ccc|cc}
\hline $\mathrm{CO}_{2}$ limit $(\mathrm{ppm})$ & 650 & & 600 & & 550 & 500 & \\
& $\gamma_{1}$ & $\varepsilon_{1 T}$ & $\gamma_{1}$ & $\varepsilon_{1 T}$ & $\gamma_{1}$ & $\varepsilon_{1 T}$ & $\gamma_{1}$ & $\varepsilon_{1 T}$ \\
\hline 600 & 0.00255 & 0.00012 & & & & & & \\
550 & 0.00351 & 0.00011 & 0.01054 & 0.00034 & & & & \\
500 & 0.00517 & 0.00011 & 0.01260 & 0.00032 & 0.01764 & 0.00050 & & \\
450 & 0.00859 & 0.00013 & 0.01870 & 0.00036 & 0.02480 & 0.00052 & 0.03701 & 0.00107 \\
\hline
\end{tabular}

risk preferences can be represented by functions in the sets $\left(V_{1} \times U_{1}\right)\left(\gamma_{1}\right)$ and $U_{1}\left(\varepsilon_{1 T}\right)$. Moreover we can say that those who do not prefer the abatement policies have an extreme combination of time and risk preferences. Violation of First-order Time and Second-order Stochastic Dominance is also on the whole very small, and note that the condition on $D_{1}^{2}(b, T)$ in Definition 3 - equivalently $H_{1}^{-1,2}(p, T) \geq 0$ - is met by all policies. The overall violation increases with the stringency of the policy.

Let us now use TSD analysis to compare the various abatement policies with each other. We know from the analysis above that exact TSD will not exist either to a first order or to a second order with respect to SD. Therefore we can proceed directly to analysing violations. In doing so we confine our attention to the least restrictive first-order TSD, given the wealth of pairwise comparisons that could potentially be made. Table 3 presents the results, in terms of violations of exact First-order TSD. The table should be read such that $F_{1}^{1}$ is the $\mathrm{CO}_{2}$ limit in the first column and $G_{1}^{1}$ is the limit in the top row. So, for example, $\gamma_{1}=0.00859$ is the violation of exact First-order TSD for $\overline{M^{A T}}=450$ over $\overline{M^{A T}}=650$.

Although we might have expected the violations to be relatively large, since the abatement policy controls are much more similar to each other than they are to BAU - and they do tend to be higher than in the comparison with BAU - in fact they are all relatively small in absolute terms, such that for any pair of policies the lower $\mathrm{CO}_{2}$ limit in the pair is almost dominant. Therefore we can go further and say that there exists a broad space for agreement, represented by everyone whose preferences are in the set $\left(V_{1} \times U_{1}\right)\left(\gamma_{1}\right)$, for tough emissions reduction targets, as tough as $\overline{M^{A T}}=450$. 


\section{How DICE yields these results}

The topography of the panels in Figure 2 tells us much about the effect of emissions abatement on consumption per capita in DICE, how this effect is related to time, and the nature of the uncertainty about the effect. In this century we can see it is often the case that $H_{1}^{-1,1}<0$, but the surface appears flat as there is little difference between the cumulative distributions. In the next century, however, the surface rises to a peak at high quantiles, revealing that the mitigation policies can yield much higher consumption per capita than BAU, albeit there is much uncertainty about whether this will eventuate and there is only a low probability associated with it. Comparing the policies, we can see that it is more likely that $H_{1}^{-1,1}<0$, the more stringent is the limit on the atmospheric stock of $\mathrm{CO}_{2}$. However, what Figure 2 does not show, due to truncating the vertical axes in order to obtain a better resolution on the boundary between

$H_{1}^{-1,1}<0$ and $H_{1}^{-1,1} \geq 0$, is that conversely the peak difference in consumption per capita is higher, the more stringent is the concentration limit.

What lies behind these patterns? In fact, Figure 2 can be seen as a new expression of a well known story about the economics of climate mitigation. There are two different sources of violation of First-order TSD. The first is that, in early years, the climate is close to its initial, relatively benign state, yet significant investment is required in emissions abatement. This makes it rather likely that consumption per capita will initially be lower under a mitigation policy than it is under BAU. The second source of violation is productivity growth, a large source of uncertainty affecting BAU consumption per capita and all that depends on it. In particular, when the realisation of the random productivity-growth parameter is at its lowest, consumption per capita is also at its lowest, moreover in these contingencies carbon emissions are very low. In these circumstances even mild emissions reductions are net costly. This latter effect is therefore isolated in Figure 2 where $\overline{M^{A T}}=650$.

On the other hand, in later years the BAU atmospheric stock of $\mathrm{CO}_{2}$ is high, so the possibility opens up that emissions abatement will deliver higher consumption per capita. How much higher depends in the main on how much damage is caused by high atmospheric $\mathrm{CO}_{2}$ and therefore how much damage can be avoided by emissions abatement. In our version of DICE this is highly uncertain - much more so than the cost of emissions abatement - and depends principally on the climate sensitivity and the damage function coefficient $\tilde{\alpha}_{3}$ in (3). It is here that the driving force can be found behind the tiny violations of exact TSD in Table 2, namely the small possibility, in the second half of the modelling horizon, that the mitigation policies will deliver much higher consumption per capita than business as usual. This is consistent with the observation in previous, related research that the tails of the distribution are critical in determining the benefits of emissions abatement (e.g. Dietz, 2011; Weitzman, 2009). 


\section{Conclusions}

In this paper we have proposed a theory of Time-Stochastic Dominance for ordering risky, intertemporal prospects. Our theory is built by unifying the insights of Stochastic Dominance (SD) and Time Dominance (TD). Like these earlier theories, the approach is non-parametric and allows orderings to be constructed only on the basis of partial information about preferences. But our approach generalises the application of simple SD to intertemporal prospects, by permitting pure temporal preferences, just as it generalises the application of simple TD to risky prospects, by avoiding the need to make strong assumptions about the characteristics of the prospects (prospects may belong to different risk classes and cashflows may be large/non-marginal).

Like other dominance criteria, a possible practical disadvantage of (exact) TSD is that it may not exist in the data, despite one prospect paying out more than another most of the time, in most states of nature. Various approaches can be taken to dealing with this. Our choice has been to extend the notion of Almost SD pioneered by Levy and others to our bi-dimensional time-risk setup, giving rise to Almost TSD.

The theory can in principle be applied to any investment problem involving multiple time-periods and uncertainty about pay-offs, however, given the involving nature of the analysis, it might prove most useful in highly contentious public-investment decisions, where there is disagreement about appropriate rates of discount and risk aversion. A leading example might be the mitigation of climate change, so we applied the theory to this policy controversy using a stochastic version of the DICE model, in which eight key model parameters were randomised and Monte Carlo simulation was undertaken.

We were unable to establish exact TSD in the data, even when moving to Second-order Stochastic Dominance (with First-order Time Dominance). However, when we analyse the related theory of Almost TSD we find that the volume/area of violation of exact TSD is generally very small indeed, so that we can say that almost all decision-makers would indeed favour any of our mitigation policies over BAU, and moreover that they would favour tougher mitigation policies over slacker alternatives. So the space for agreement is large in this regard.

Clearly our empirical results depend on the structure of the DICE model and how we have parameterised it; our approach is only non-parametric as far as preferences are concerned. Of particular note are the key roles played by uncertainty about climate sensitivity, the curvature of the damage function, and productivity growth. Our parameterisation of the former two is key in producing a small violation of exact TSD, because when a high climate sensitivity combines with a high curvature on the damage function, the difference in the relevant cumulative pay-off distributions becomes very large. Our parameterisation of initial TFP growth, specifically our assumption via an unbounded normal distribution that it could be very low or even negative over long periods, is conversely key in producing a violation in the first place. It will be very interesting to see what results are obtained with different integrated assessment 
models, or with different implementations of DICE.

Our interpretation of $\gamma_{k}, \varepsilon_{k T}$ and $\lambda_{1 b}$ in the application of Almost TSD is also open to debate, given the nature of the concept. Research on almost dominance relations is still at a relatively early stage, so we lack data on the basis of which we can say with high confidence that some preferences are extreme, while others are not. Nonetheless our violations are for the most part so small that we are somewhat immune to this criticism.

\section{References}

Arrow, K. J., Cline, W., Maler, K., Munasinghe, M., Squitieri, R., Stiglitz, J., 1996. Intertemporal equity, discounting, and economic efficiency. In: IPCC (Ed.), Climate Change 1995: Economic and Social Dimensions of Climate Change. Cambridge University Press, pp. 127-144.

Atkinson, A. B., Bourguignon, F., 1982. The comparison of multi-dimensioned distributions of economic status. The Review of Economic Studies 49 (2), $183-201$.

Bøhren, Ø., Hansen, T., 1980. Capital budgeting with unspecified discount rates. The Scandinavian Journal of Economics, 45-58.

Clarke, L., Jiang, K., Akimoto, K., M., B., Blanford, G., Fisher-Vanden, K., Hourcade, J.-C., Krey, V., Kriegler, E., Löschel, A., McCollum, D., Paltsev, S., Rose, S., Shukla, P. R., Tavoni, M., van der Zwaan, B. C. C., van Vuuren, D., 2014. Asessing transformation pathways. In: Edenhofer, O., PichsMadruga, R., Sokona, Y., Farahani, E., Kadner, S., Seyboth, K., Adler, A., Baum, I., Brunner, S., Eickemeier, P., Kriemann, B., Savolainen, J., Schlömer, S., von Stechow, C., Zwickel, T., Minx, J. (Eds.), Climate Change 2014: Mitigation of Climate Change. Contribution of Working Group III to the Fifth Assessment Report of the Intergovernmental Panel on Climate Change. Cambridge University Press, Cambridge, UK and New York, NY, USA.

Cline, W. R., 1992. The Economics of Global Warming. Peterson Institute.

Dasgupta, P., 2007. The stern review's economics of climate change. National Institute Economic Review 199 (1), 4-7.

Dietz, S., 2011. High impact, low probability? an empirical analysis of risk in the economics of climate change. Climatic Change 103 (3), 519-541.

Dietz, S., Asheim, G., 2012. Climate policy under sustainable discounted utilitarianism. Journal of Environmental Economics and Management 63, 321335 .

Dietz, S., Hepburn, C. J., Forthcoming. Benefit-cost analysis of non-marginal climate and energy projects. Energy Economics. 
Ekern, S., 1981. Time dominance efficiency analysis. The Journal of Finance 36 (5), 1023-1033.

Fishburn, P. C., 1964. Decision and Value Theory. Wiley.

Fishburn, P. C., Vickson, R. G., 1978. Theoretical foundations of stochastic dominance. In: Whitmore, G. A., Findlay, M. C. (Eds.), Stochastic Dominance: an Approach to Decision-Making under Risk. Lexington Books, pp. $39-113$.

Gollier, C., 2006. An evaluation of stern's report on the economics of climate change.

Hadar, J., Russell, W. R., 1969. Rules for ordering uncertain prospects. American Economic Review 59 (1), 25-34.

Hanoch, G., Levy, H., 1969. The efficiency analysis of choices involving risk. Review of Economic Studies 36 (3), 335-346.

Hepburn, C. J., Beckerman, W., 2007. Ethics of the discount rate in the stern review on the economics of climate change. World Economics 8 (1).

Ikefuji, M., Laeven, R. J., Magnus, J. R., Muris, C., 2012. Pareto utility. Theory and Decision, 1-15.

Karcher, T., Moyes, P., Trannoy, A., 1995. The stochastic dominance ordering of income distributions over time: The discounted sum of the expected utilities of incomes. In: Barnett, W. A., Moulin, H., Salles, M., Schofield, N. J. (Eds.), Social Choice, Welfare, and Ethics: Proceedings of the Eighth International Symposium in Economic Theory and Econometrics. Cambirgde University Press.

Kelly, D. L., Kolstad, C. D., 1999. Bayesian learning, growth, and pollution. Journal of economic dynamics and control 23 (4), 491-518.

Kimball, M. S., 1990. Precautionary saving in the small and in the large. Econometrica, 53-73.

Lemoine, D., Traeger, C., 2014. Watch your step: optimal policy in a tipping climate. American Economic Journal: Economic Policy 6 (1), 137-166.

Leshno, M., Levy, H., 2002. Preferred by all and preferred by most decision makers: Almost stochastic dominance. Management Science 48 (8), 10741085 .

Levy, H., 1973. Stochastic dominance, efficiency criteria, and efficient portfolios: The multi-period case. The American Economic Review 63 (5), 986-994.

Levy, H., Hanoch, G., 1970. Relative effectiveness of efficiency criteria for portfolio selection. Journal of Financial and Quantitative Analysis, 63-76. 
Levy, H., Kroll, Y., 1979. Efficiency analysis with borrowing and lending: criteria and their effectiveness. The Review of Economics and Statistics 61 (1), $125-130$.

Levy, H., Leshno, M., Leibovitch, B., 2010. Economically relevant preferences for all observed epsilon. Annals of Operations Research 176 (1), 153-178.

Levy, H., Paroush, J., 1974a. Multi-period stochastic dominance. Management Science 21 (4), 428-435.

Levy, H., Paroush, J., 1974b. Toward multivariate efficiency criteria. Journal of Economic Theory 7 (2), 129-142.

Levy, M., 2009. Almost stochastic dominance and stocks for the long run. European Journal of Operational Research 194 (1), 250-257.

Meehl, G., Stocker, T., Collins, W., Friedlingstein, A., Gaye, A., Gregory, J., Kitoh, A., Knutti, R., Murphy, J., Noda, A., Raper, S., Watterson, I., Weaver, A., Zhao, Z.-C., 2007. Global climate projections. In: Solomon, S., Qin, D., Manning, M., Chen, Z., Marquis, M., Averyt, K., Tignor, M., Miller, H. (Eds.), Climate Change 2007: The Physical Science Basis. Contribution of Working Group I to the Fourth Assessment Report of the Intergovernmental Panel on Climate Change. Cambridge University Press, Cambridge, UK.

Nordhaus, W. D., 1991. To slow or not to slow: the economics of the greenhouse effect. The Economic Journal 101 (407), 920-937.

Nordhaus, W. D., 1993a. Optimal greenhouse-gas reductions and tax policy in the "dice" model. The American Economic Review 83 (2), 313-317.

Nordhaus, W. D., 1993b. Rolling the "dice": an optimal transition path for controlling greenhouse gases. Resource and Energy Economics 15 (1), 27-50.

Nordhaus, W. D., 1994. Managing the Global Commons: the Economics of Climate Change. MIT Press, Cambridge, MA.

Nordhaus, W. D., 2007. A review of the "stern review on the economics of climate change". Journal of Economic Literature, 686-702.

Nordhaus, W. D., 2008. A Question of Balance: Weighing the Options on Global Warming Policies. Yale University Press.

Pindyck, R. S., 2011. Fat tails, thin tails, and climate change policy. Review of Environmental Economics and Policy 5 (2), 258-274.

Roe, G., Baker, M., 2007. Why is climate sensitivity so unpredictable? Science 318 (5850), 629-632.

Rothschild, M., Stiglitz, J. E., 1970. Increasing risk: I. a definition. Journal of Economic Theory 2 (3), 225-243. 
Scarsini, M., 1986. Comparison of random cash flows. IMA Journal of Management Mathematics 1 (1), 25-32.

Stern, N., 2007. The Economics of Climate Change: the Stern Review. Cambridge University Press.

Stern, N., 2008. The economics of climate change. The American Economic Review: Papers and Proceedings 98 (2), 1-37.

Tol, R. S. J., 2012. On the uncertainty about the total economic impact of climate change. Environmental and Resource Economics 53 (1), 97-116.

Tzeng, L. Y., Huang, R. J., Shih, P.-T., 2012. Revisiting almost second-degree stochastic dominance. Management Science.

Weitzman, M., 2007. A review of the Stern Review on the economics of climate change. Journal of Economic Literature 45 (3), 703-724.

Weitzman, M., 2009. On modeling and interpreting the economics of catastrophic climate change. The Review of Economics and Statistics 91 (1), 1-19.

Weitzman, M., 2012. Ghg targets as insurance against catastrophic climate damages. Journal of Public Economic Theory 14 (2), 221-244. 


\section{Annex 1}

\section{A primer on Stochastic Dominance and 'Almost' Stochastic Dominance}

Stochastic Dominance (SD) determines the order of preference of an expectedutility maximiser between risky prospects, while requiring minimal knowledge of her utility function. Take any two risky prospects with probability distributions $F$ and $G$ respectively and denote their cumulative distributions $F^{1}$ and $G^{1}$ respectively. Assuming the cumulative distributions have finite support on $[a, b]$, $F$ is said to first-order stochastic dominate $G$ if and only if $F^{1}(x) \leq G^{1}(x), \forall x \in$ $[a, b]$ and there is a strict inequality for at least one $x$, where $x$ is a realisation from the distribution of pay-offs possible from a prospect. Moreover it can be shown that any expected-utility maximiser with a utility function belonging to the set of non-decreasing utility functions $U_{1}=\left\{u: u^{\prime}(x) \geq 0\right\}$ would prefer $F$.

First-order SD does not exist if the cumulative distributions cross, which means that, while it is a powerful result in the theory of choice under uncertainty, the practical usefulness of the theorem is limited. By contrast, where $F^{2}(x)=$ $\int_{a}^{x} F^{1}(s) d s$ and $G^{2}(x)=\int_{a}^{x} G^{1}(s) d s, F$ second-order stochastic dominates $G$ if and only if $F^{2}(x) \leq G^{2}(x), \forall x \in[a, b]$ and there is a strict inequality for at least one $x$. It can be shown that any expected-utility maximiser with a utility function belonging to the set of all non-decreasing and (weakly) concave utility functions $U_{2}=\left\{u: u \in U_{1}\right.$ and $\left.u^{\prime \prime}(x) \leq 0\right\}$ would prefer $F$, i.e. any such (weakly) risk-averse decision-maker. Hence second-order SD can rank inter alia prospects with the same mean but different variances.

Nonetheless the practical usefulness of second-order SD is still limited, as the example in the Introduction illustrated. One could proceed by placing an additional restriction on the decision-maker's preferences, defining the set $U_{3}=\left\{u: u \in U_{2}\right.$ and $\left.u^{\prime \prime \prime}(x) \geq 0\right\}$ and looking for third-order SD. Decisionmakers exhibiting decreasing absolute risk aversion have preferences represented by utility functions in $U_{3}$ and such decision-makers will also exhibit 'prudence' in inter-temporal savings decisions (Kimball, 1990). Where $F^{3}(x)=\int_{a}^{x} F^{2}(s) d s$ and $G^{3}(x)=\int_{a}^{x} G^{2}(s) d s, F$ third-order stochastic dominates $G$ if and only if $F^{3}(x) \leq G^{3}(x), \forall x \in[a, b]$ and $E_{F}(x) \geq E_{G}(x)$, and there is at least one strict inequality. However, it can easily be verified in the example that $G^{3}(x)-F^{3}(x)<0, x \in[0.5,1)$, yet $E_{F}(x)>>E_{G}(x)$, so third-order SD does not exist. Moreover SD cannot be established to any order in this example, because the first non-zero values of $G^{1}(x)-F^{1}(x)$ are negative as $x$ increases from its lower bound, yet $E_{F}(x)>E_{G}(x)$. Successive rounds of integration will not make this go away.

A more fruitful route is the theory of Almost SD set out by Leshno and Levy (2002) and recently further developed by Tzeng et al. (2012). Almost SD places restrictions on the derivatives of the utility function with the purpose of excluding the extreme preferences that prevent exact SD from being established. Dominance relations are then characterised for 'almost' all decision-makers.

For every $0<\varepsilon_{k}<0.5$, where $k=1,2$ corresponds to first- and second-order 
SD respectively, define subsets of $U_{k}$ :

$$
\begin{aligned}
& U_{1}\left(\varepsilon_{1}\right)=\left\{u \in U_{1}: \frac{u^{\prime}(x)}{\inf \left[u^{\prime}(x)\right]} \leq\left[\frac{1}{\varepsilon_{1}}-1\right], \forall x\right\} \text { and } \\
& U_{2}\left(\varepsilon_{2}\right)=\left\{u \in U_{2}: \frac{-u^{\prime \prime}(x)}{\inf \left[-u^{\prime \prime}(x)\right]} \leq\left[\frac{1}{\varepsilon_{2}}-1\right], \forall x\right\} .
\end{aligned}
$$

$U_{1}\left(\varepsilon_{1}\right)$ is the set of non-decreasing utility functions with the added restriction that the ratio between maximum and minimum marginal utility is bounded by $\frac{1}{\varepsilon_{1}}-1$. In the limit as $\varepsilon_{1}$ approaches 0.5 , the only function in $U_{1}\left(\varepsilon_{1}\right)$ is linear utility. Conversely as $\varepsilon_{1}$ approaches zero, $U_{1}\left(\varepsilon_{1}\right)$ coincides with $U_{1} . U_{2}\left(\varepsilon_{2}\right)$ is the set of non-decreasing, weakly concave utility functions with an analogous restriction on the ratio between the maximum and minimum values of $u^{\prime \prime}(x)$. In the limit as $\varepsilon_{2}$ approaches $0.5, U_{2}\left(\varepsilon_{2}\right)$ contains only linear and quadratic utility functions, while as $\varepsilon_{2}$ approaches zero, it coincides with $U_{2}$.

Defining the set of realisations over which exact first-order SD is violated as

$$
S^{1}(F, G)=\left\{x \in[a, b]: G^{1}(x)<F^{1}(x)\right\},
$$

$F$ is said to first-order almost stochastic dominate $G$ if and only if

$$
\int_{S^{1}}\left[F^{1}(x)-G^{1}(x)\right] d x \leq \varepsilon_{1} \cdot \int_{a}^{b}\left|\left[F^{1}(x)-G^{1}(x)\right]\right| d x .
$$

Moreover, in a similar vein to exact SD, it can be shown that any expectedutility maximiser with a utility function belonging to $U_{1}\left(\varepsilon_{1}\right)$ would prefer $F$.

Defining the set of realisations over which exact second-order SD is violated as

$$
S^{2}(F, G)=\left\{x \in[a, b]: G^{2}(s)<F^{2}(s)\right\},
$$

$F$ second-order almost stochastic dominates $G$ if and only if

$$
\begin{gathered}
\int_{S^{2}}\left[F^{2}(x)-G^{2}(x)\right] d x \leq \varepsilon_{2} \cdot \int_{a}^{b}\left|\left[F^{2}(x)-G^{2}(x)\right]\right| d x \text { and } \\
E_{F}(x) \geq E_{G}(x) .
\end{gathered}
$$

Any expected-utility maximiser with a utility function belonging to $U_{2}\left(\varepsilon_{2}\right)$ would prefer $F$. From these definitions of first- and second-order Almost SD one can see that $\varepsilon_{k}$ intuitively represents the proportion of the total area between $F^{k}$ and $G^{k}$ in which the condition for exact SD of the $\mathrm{k}^{t h}$ order is violated. The smaller is $\varepsilon_{k}$, the smaller is the relative violation.

\section{A primer on Time Dominance}

The theory of Time Dominance (TD) builds on the SD approach to choice problems under uncertainty, and transfers it to problems of intertemporal choice (Bøhren and Hansen, 1980; Ekern, 1981). Denoting the cumulative cashflows of 
any two investments $X_{1}$ and $Y_{1},{ }^{14} X$ is said to first-order time dominate $Y$ if and only if $X_{1}(t) \geq Y_{1}(t), \forall t \in[0, T]$ and there is a strict inequality for some $t$, where $T$ is the terminal period of the most long-lived project. Moreover it can be shown that any decision-maker with a discount function belonging to the set of all decreasing consumption discount functions $\widehat{V_{1}}=\left\{\widehat{v}: \widehat{v}^{\prime}(t)<0\right\}$ would prefer $X$. Thus if the decision-maker prefers a dollar today to a dollar tomorrow, she will prefer $X$ if it first-order time dominates $Y$.

Just like SD, first-order TD has limited practical purchase, because the set of undominated investments remains large, i.e. the criterion $X_{1}(t) \geq Y_{1}(t), \forall t$ is restrictive. ${ }^{15}$ Therefore, proceeding again by analogy to SD, $X$ second-order time dominates $Y$ if and only if

$$
\begin{gathered}
X_{1}(T) \geq Y_{1}(T) \text { and } \\
X_{2}(t) \geq Y_{2}(t), \forall t \in[0, T],
\end{gathered}
$$

where $X_{2}(t)=\int_{0}^{t} X_{1}(\tau) d \tau$ and $Y_{2}(t)=\int_{0}^{t} Y_{1}(\tau) d \tau$, and there is at least one strict inequality. Any decision-maker with a discount function belonging to the set of all decreasing, convex consumption discount functions $\widehat{V_{2}}=\{\widehat{v}: \widehat{v} \in$ $\widehat{V_{1}}$ and $\left.\widehat{v}^{\prime \prime}(t)>0\right\}$ would prefer $X$. This set includes exponential discounting. Noting how the conditions for second-order TD are obtained from their counterparts for first-order TD by integration, TD can be defined to the $\mathrm{n}^{\text {th }}$ order (see Ekern, 1981).

Notice that TD applies to deterministic cashflows. It would be possible to apply the method to uncertain cashflows, if $X$ and $Y$ were expected cashflows and if a corresponding risk adjustment were made to $\{\widehat{v}\}$. However, since any two cashflows $X$ and $Y$ would then be discounted using the same set of riskadjusted rates, it would be necessary to assume that the cashflows belong to the same risk class (Bøhren and Hansen, 1980), for example under the capital asset pricing model they would have to share the same covariance with the market portfolio. This significantly limits the reach of the method to uncertain investments. It would also be necessary to assume that any investments being compared are small (i.e. marginal), since the domain of $\{\widehat{v}\}$ is cashflows and therefore depends on a common assumed growth rate. Neither of these assumptions is likely to hold in the case of climate change (see Weitzman, 2007, for a discussion of the covariance between climate mitigation and market returns, and Dietz and Hepburn, Forthcoming, for a discussion of whether climate mitigation is non-marginal).

\footnotetext{
${ }^{14} X_{1}(t)=\int_{0}^{t} x(\tau) d \tau$ and $Y_{1}(t)=\int_{0}^{t} y(\tau) d \tau$.

${ }^{15}$ Indeed, in the domain of deterministic cashflows over multiple time-periods, the requirement that $X^{1}(0) \geq Y^{1}(0)$ means that one investment cannot dominate another by a first, second or higher order, if the initial cost is higher, no matter what the later benefits are. This makes it difficult to compare investments of different sizes. However, this can be worked around by normalising the cashflows to the size of the investment (Bøhren and Hansen, 1980).
} 


\section{Annex 2}

\section{Proof of Proposition 1}

\section{Sufficiency:}

We want to prove that

$$
\begin{array}{ccc}
D_{1}^{1}(z, t) \geq 0 & \Longrightarrow & N P V_{v, u}(X) \geq N P V_{v, u}(Y) \\
\text { for all } t \text { and } z & & \text { for all } u \in U_{1}, v \in V_{1} .
\end{array}
$$

Assume that $z$ is bounded from below and above, $a \leq z \leq b$. This implies that for $z<a, F^{1}(x, t)=G^{1}(y, t)=D^{1}(z, t)=0$ for all $t \in[0, T]$, while similarly for $z>b, D^{1}(z, t)=G^{1}(y, t)-F^{1}(x, t)=1-1=0$ for all $t \in[0, T]$.

Denote by

$$
\begin{aligned}
\Delta & \equiv N P V_{v, u}(X)-N P V_{v, u}(Y)=\int_{0}^{T} v(t) E_{F} u(x) d t-\int_{0}^{T} v(t) E_{G} u(y) d t \\
& =\int_{0}^{T} v(t) \int_{a}^{b} f(x, t) u(x) d x d t-\int_{0}^{T} v(t) \int_{a}^{b} g(y, t) u(y) d y d t \\
& =\int_{0}^{T} v(t) \int_{a}^{b}-d(z, t) u(z) d z d t .
\end{aligned}
$$

Integrating by parts with respect to $z$ we obtain

$$
\Delta=\int_{0}^{T} v(t)\left[\left.u(z)(-) D^{1}(z, t)\right|_{a} ^{b}-\int_{a}^{b}(-) D^{1}(z, t) u^{\prime}(z) d z\right] d t
$$

The first term in the square brackets is equal to zero (recall that for $z=b$, we have $D^{1}(b, t)=1-1=0$ for all $t$ and for $z=a$ we have $D^{1}(a, t)=0$ for all $t$ ). Therefore, we are left with

$$
\begin{aligned}
\Delta & =\int_{0}^{T} v(t)\left[-\int_{a}^{b}(-) D^{1}(z, t) u^{\prime}(z) d z\right] d t \\
& =\int_{0}^{T} \int_{a}^{b} v(t) D^{1}(z, t) u^{\prime}(z) d z d t .
\end{aligned}
$$

Integrating by parts with respect to $t$ we have

$$
\begin{aligned}
\Delta & =\int_{a}^{b}\left[\left.D_{1}^{1}(z, t) v(t)\right|_{0} ^{T}-\int_{0}^{T} D_{1}^{1}(z, t) v^{\prime}(t) d t\right] u^{\prime}(z) d z \\
& =\int_{a}^{b}\left[D_{1}^{1}(z, T) v(T)-\int_{0}^{T} D_{1}^{1}(z, t) v^{\prime}(t) d t\right] u^{\prime}(z) d z,
\end{aligned}
$$

as $D_{1}^{1}(z, 0)=G_{1}^{1}(y, 0)-F_{1}^{1}(x, 0)=0$ for all $z \in[a, b]$.

From our initial assumption about the bounding of $z$, we know that $D_{1}^{1}(z, t) \geq$ 0 and $v(T) \geq 0$. Hence for all $u \in U_{1}$ and $v \in V_{1}, N P V_{v, u}(X) \geq N P V_{v, u}(Y)$. 
Necessity:

We have to prove that

$$
\begin{array}{ccc}
N P V_{v, u}(X) \geq N P V_{v, u}(Y) & \Longrightarrow & D_{1}^{1}(z, t) \geq 0 \\
\text { for all } u \in U_{1}, v \in V_{1} & \text { for all } t \text { and } z .
\end{array}
$$

Starting from Eq. (4), let $(\widetilde{z}, \widetilde{t})$ be the smallest (in the lexicographic sense) pair $(z, t)$ such that $D_{1}^{1}(\widetilde{z}, \widetilde{t})<0$. We will show that there is a utility function $\widetilde{u} \in U_{1}$ and a discount function $\widetilde{v} \in V_{1}$ for which our supposition implies that $N P V_{v, u}(X)<N P V_{v, u}(Y)$, thus contradicting the original assumption.

Supposing that a violation $D_{1}^{1}(\widetilde{z}, \widetilde{t})<0$ does exist, since $D_{1}^{1}$ is continuous it will also exist in the range $\widetilde{z} \leq z \leq \widetilde{z}+\varepsilon$. Define the following utility function:

$$
\widetilde{u}(z)=\left\{\begin{array}{cc}
\widetilde{z} & z<\widetilde{z} \\
z & \widetilde{z} \leq z \leq \widetilde{z}+\varepsilon \\
\widetilde{z}+\varepsilon & z>\widetilde{z}+\varepsilon
\end{array}\right.
$$

noting that $\widetilde{u}(z) \notin U_{1}$, strictly speaking, but that it can be approximated arbitrarily closely by a function that does belong to $U_{1}$ (see Fishburn and Vickson 1978, p. 75).

Similarly define the following discount function:

$$
\widetilde{v}(t)=\left\{\begin{array}{lr}
1+p e^{-p t} & \text { if } 0 \leq t \leq \tilde{t} \\
0+p e^{-p t} & \widetilde{t}<t \leq T
\end{array}\right.
$$

which again is discontinuous but can be approximated arbitrarily closely by some $\widetilde{v} \in V_{1}$.

Substituting these functions into (4) we obtain

$$
\begin{aligned}
\Delta & =\int_{\widetilde{z}}^{\tilde{z}+\varepsilon}\left[\int_{0}^{\widetilde{t}} D^{1}(x, t) d t+p \int_{0}^{T} e^{-p t} D^{1}(x, t) d t\right] d z \\
& =\int_{\widetilde{z}}^{\widetilde{z}+\varepsilon}\left[\left.D_{1}^{1}(z, t)\right|_{0} ^{\widetilde{t}}+p \int_{0}^{T} e^{-p t} D^{1}(z, t) d t\right] d z \\
& =\int_{\widetilde{z}}^{\widetilde{z}+\varepsilon}\left[D_{1}^{1}(z, \widetilde{t})+p \int_{0}^{T} e^{-p t} D^{1}(z, t) d t\right] d z
\end{aligned}
$$

In the limit as $p \rightarrow 0, p \int_{0}^{T} e^{-p t} D^{1}(z, t) d t=0$, therefore for a sufficiently small $p, D_{1}^{1}(\widetilde{z}, \widetilde{t})<0$ implies that $N P V_{v, u}(X)<N P V_{v, u}(Y)$, contradicting the initial assumption and showing it is necessary that $D_{1}^{1}(\widetilde{z}, \widetilde{t}) \geq 0$ for all $z \in[a, b]$ and $t \in[0, T]$.

\section{Proof of Proposition 2}

We need to prove that the following holds:

$$
\begin{aligned}
& H_{1}^{-1}(p, t)=F_{1}^{-1}(p, t)-G_{1}^{-1}(p, t) \geq 0, \quad \Longleftrightarrow \quad D_{1}^{1}(z, t)=G_{1}^{1}(z, t)-F_{1}^{1}(z, t) \geq 0 \\
& \forall p \in[0,1] \text { and } t \in[0, T] \quad \forall z \in[a, b] \text { and } \forall t \in[0, T]
\end{aligned}
$$


Given that $F_{1}^{1}(z, t) \leq G_{1}^{1}(z, t)$ is an optimal decision rule for all $(v, u) \in V_{1} \times U_{1}$, if the above expression holds, so will $F_{1}^{-1}(p, t) \geq G_{1}^{-1}(p, t)$.

Assume first that $F_{1}^{1}(z, t) \leq G_{1}^{1}(z, t)$ for all $z \in[a, b]$ and all $t \in[0, T]$. This means that for an arbitrary $x^{*}(t)$ we have $F_{1}^{1}\left(x^{*}, t\right)=p_{1}^{*}(t) \leq G_{1}^{1}\left(x^{*}, t\right)=p_{2}^{*}(t)$. In this way, for given $t x^{*}$ will represent both the $p_{1}^{* t h}$ quantile of distribution $F$ and the $p_{2}^{* t h}$ quantile of distribution $G$.

Since, by assumption, $F$ and $G$ are monotonic increasing functions of $z$, the quantile functions are monotonic increasing functions of $p \in[0,1]$. Therefore, knowing that $p_{1}^{*}(t) \leq p_{2}^{*}(t)$ and due to the monotonicity of the quantile function, $G_{1}^{-1}\left(p_{1}^{*}, t\right) \leq G_{1}^{-1}\left(p_{2}^{*}, t\right)$. Remembering that $x^{*}(t)=G_{1}^{-1}\left(p_{2}^{*}, t\right)=F_{1}^{-1}\left(p_{1}^{*}, t\right)$, it follows that $G_{1}^{-1}\left(p_{1}^{*}, t\right) \leq F_{1}^{-1}\left(p_{1}^{*}, t\right)$.

We conclude that, for every $t \in[0, T]$, the condition $F_{1}^{1}(z, t) \leq G_{1}^{1}(z, t), \forall z \in$ $[a, b]$ implies $F_{1}^{-1}(p, t) \geq G_{1}^{-1}(p, t) \forall p$. The analogous logic can be applied to show the reverse condition also holds, that is for a given $t, F_{1}^{-1}(p, t) \geq G_{1}^{-1}(p, t)$ will imply $F_{1}^{1}(z, t) \leq G_{1}^{1}(z, t)$.

\section{Proof of Proposition 3}

\section{Sufficiency:}

Starting with the expression derived in the previous proof

$$
\Delta=\int_{0}^{T} v(t) \int_{a}^{b} u^{\prime}(z) D^{1}(z, t) d z d t
$$

we continue by integrating again with respect to $z$ :

$$
\begin{aligned}
\Delta & =\int_{0}^{T} v(t)\left[\left.u^{\prime}(z) D^{2}(z, t)\right|_{a} ^{b}-\int_{a}^{b} u^{\prime \prime}(z) D^{2}(z, t) d z\right] d t \\
& =\int_{0}^{T} v(t) u^{\prime}(b) D^{2}(b, t) d t-\int_{0}^{T} v(t) \int_{a}^{b} u^{\prime \prime}(z) D^{2}(z, t) d z d t
\end{aligned}
$$

Now integrating by parts with respect to time $t$,

$$
\begin{aligned}
\Delta= & \left.u^{\prime}(b) v(t) D_{1}^{2}(b, t)\right|_{0} ^{T}-u^{\prime}(b) \int_{0}^{T} v^{\prime}(t) D_{1}^{2}(b, t) d t- \\
& -\left.\int_{a}^{b} u^{\prime \prime}(z) v(t) D_{1}^{2}(z, t)\right|_{0} ^{T}+\int_{a}^{b} u^{\prime \prime}(z) \int_{0}^{T} v^{\prime}(t) D_{1}^{2}(z, t) d t d z . \\
\Delta= & u^{\prime}(b) v(T) D_{1}^{2}(b, T)-u^{\prime}(b) \int_{0}^{T} v^{\prime}(t) D_{1}^{2}(b, t) d t- \\
& -\int_{a}^{b} u^{\prime \prime}(z) v(T) D_{1}^{2}(z, T) d z+\int_{a}^{b} u^{\prime \prime}(z) \int_{0}^{T} v^{\prime}(t) D_{1}^{2}(z, t) d t d z .
\end{aligned}
$$


From this last expression we can extract the conditions for dominance with respect to $V_{1} \times U_{2}$ presented in Proposition 3. That is, $D_{1}^{2}(z, t) \geq 0$ for all $z \in[a, b]$ and all $t \in[0, T]$ is a sufficient condition for $N P V_{v, u}(X) \geq N P V_{v, u}(Y)$ for all $\{v, u\} \in V_{1} \times U_{2}$.

Necessity:

Consider the increasing and concave utility function defined by

$$
\widetilde{u}(z):= \begin{cases}z-\widetilde{z} & \text { for } a \leq z<\widetilde{z} \\ 0 & \text { for } \widetilde{z} \leq z \leq b\end{cases}
$$

and let $\widetilde{U} \in U_{2}$ be a suitable approximation of $\widetilde{u}$. The proofs of necessity are similar to the proofs of necessity of the previous proposition and are therefore omitted.

\section{Proof of Proposition 5}

Sufficiency:

We want to prove that

$$
\begin{array}{ll} 
& X>_{A 1 T S} Y \\
\Rightarrow \quad & N P V_{v, u}(X) \geq N P V_{v, u}(Y) \\
& \forall v \in\left(V_{1} \times U_{1}\right)\left(\gamma_{1}\right) \text { and } \forall u \in U_{1}\left(\varepsilon_{1 T}\right)
\end{array}
$$

Going back to

$$
\begin{aligned}
\Delta & =\int_{a}^{b} D_{1}^{1}(z, T) v(T) d z-\int_{a}^{b} \int_{0}^{T} D_{1}^{1} v^{\prime}(t) u^{\prime}(z) d t d z \\
& =v(T) \int_{a}^{b} u^{\prime}(z) D_{1}^{1}(z, T) d z+\int_{a}^{b} \int_{0}^{T}(-) D_{1}^{1} v^{\prime}(t) u^{\prime}(z) d t d z \\
& =E+\Gamma .
\end{aligned}
$$

Separate the range $[a, b]$ at time $T$ between the part $S^{1, T}$, where $D_{1}^{1}(z, T)<0$, and the complementary part $\overline{S^{1, T}}$, where $D_{1}^{1}(z, T) \geq 0$ :

$$
\begin{aligned}
E & =v(T) \int_{a}^{b} u^{\prime}(z)\left[D_{1}^{1}(z, T)\right] d z \\
& =v(T) \int_{S^{1, T}} u^{\prime}(z) D_{1}^{1}(z, T) d z+v(T) \int_{\overline{S^{1, T}}} u^{\prime}(z) D_{1}^{1}(z, T) d z \geq 0 .
\end{aligned}
$$

Note that the integral over the range $S^{1, T}$ is negative and the integral over $\overline{S^{1, T}}$ is positive. In order for $E \geq 0$, the area where $D_{1}^{1}(z, T)<0$ must be $\varepsilon_{1 T}$ smaller than the total area enclosed between the two distributions. This restriction can be obtained from the proof of Almost First-order Stochastic Dominance by Leshno and Levy (2002), simply by requiring that the utility function belong to the subset $U_{1}\left(\varepsilon_{1 T}\right)$, where the subscript indicates that the 
bounds on maximum and minimum marginal utility are established with respect to period $T$ specifically.

Turning to $\Gamma$, separate $[a, b]$ for all $t$ into $S_{1}^{1}$, defined over ranges where $D_{1}^{1}(z, t)<0$, and $\overline{S_{1}^{1}}$, the range over which $D_{1}^{1}(z, t) \geq 0$, so that we obtain

$$
\begin{gathered}
\Gamma=\iint_{S_{1}^{1}}\left[D_{1}^{1}(z, t)\right]\left(-v^{\prime}(t) u^{\prime}(z)\right) d z d t+ \\
\iint_{\overline{S_{1}^{1}}}\left[D_{1}^{1}(z, t)\right]\left(-v^{\prime}(t) u^{\prime}(z)\right) d z d t \geq 0 .
\end{gathered}
$$

The first element of $\Gamma$ is negative and is minimised when the product of the marginals of the discount and utility functions $\left[-v^{\prime}(t) u^{\prime}(z)\right]$ is maximised, while the second element is positive and minimised when $\left[-v^{\prime}(t) u^{\prime}(z)\right]$ is minimised. Hence denoting inf $z \in[a, b] \forall t\left\{-v^{\prime}(t) u^{\prime}(z)\right\}=\underline{\theta}$ and $\sup _{z \in[a, b] \forall t}\left\{-v^{\prime}(t) u^{\prime}(z)\right\}=\bar{\theta}$, the minimum value of $\Gamma$ is

$$
\Gamma^{*}=\bar{\theta} \iint_{S_{1}^{1}}\left[D_{1}^{1}(z, t)\right] d z d t+\underline{\theta} \iint_{\overline{S_{1}^{1}}}\left[D_{1}^{1}(z, t)\right] d z d t \geq 0 .
$$

It follows that, for a given combination of discount and utility functions, $\Gamma \geq 0$ if $\Gamma^{*} \geq 0$, which can be rewritten as

$$
\left.\sup \left[-v^{\prime}(t) u^{\prime}(z)\right] \leq \inf \left[-v^{\prime}(t) u^{\prime}(z)\right)\right] \frac{\iint_{\overline{S_{1}^{1}}} D_{1}^{1}(z, t) d z d t}{\iint_{S_{1}^{1}} D_{1}^{1}(z, t) d z d t}
$$

Let $(v, u) \in\left(V_{1} \times U_{1}\right)\left(\gamma_{1}\right)$, then by definition of $\left(V_{1} \times U_{1}\right)\left(\gamma_{1}\right)$, we know that

$$
\left[-v^{\prime}(t) u^{\prime}(z)\right] \leq \sup \left[-v^{\prime}(t) u^{\prime}(z)\right] \leq \inf \left[-v^{\prime}(t) u^{\prime}(z)\right]\left[\frac{1}{\gamma_{1}}-1\right],
$$

which implies $\Gamma^{*} \geq 0$ and therefore $N P V_{v, u}(X) \geq N P V_{v, u}(Y)$.

Necessity:

Begin by assuming the opposite of necessity, i.e. that $N P V_{v, u}(X)<N P V_{v, u}(Y)$, for all functions $(v, u) \in\left(V_{1} \times U_{1}\right)\left(\gamma_{1}\right)$ and for all $u \in U_{1}\left(\varepsilon_{1 T}\right)$, implies $X>_{A 1 T S}$ $Y$. We will prove that this cannot be the case.

Suppose that

$$
\begin{aligned}
& \text { i) } \iint_{S_{1}^{1}}\left[-D_{1}^{1}(z, t)\right] d z d t>\gamma_{1} \int_{0}^{T} \int_{a}^{b}\left|D_{1}^{1}(z, t)\right| d z d t \text { and } \\
& \text { ii) } \int_{S^{1, T}}\left[-D_{1}^{1}(z, T)\right] d z>\varepsilon_{1 T} \int_{a}^{b}\left|D_{1}^{1}(z, T)\right| d z
\end{aligned}
$$

Let $\bar{\theta}$ and $\underline{\theta}$ be two positive real numbers such that $\gamma_{1}=\frac{\underline{\theta}}{\underline{\theta}+\bar{\theta}}$. Consider the pair of functions $(v, u) \in\left(V_{1} \times U_{1}\right)\left(\gamma_{1}\right)$ and where $u \in U_{1}\left(\varepsilon_{1 T}\right)$, whose product has the following properties:

$$
\begin{aligned}
& v^{\prime}(t) u(b)=0 \\
& v(T) u^{\prime}(z)=0, \\
& v^{\prime}(t) u^{\prime}(z)=-\theta \text { on } \overline{S_{1}^{1}} \text { and } \\
& v^{\prime}(t) u^{\prime}(z)=-\bar{\theta} \text { on } S_{1}^{1} .
\end{aligned}
$$


In other words, the product of $v$ and $u$ is a function proportional to

$$
v(t) u(z)=z t-b t-z T+b T .
$$

It follows then that

$$
\begin{aligned}
& N P V_{v, u}(X)-N P V_{v, u}(Y) \\
= & \bar{\theta} \iint_{S_{1}^{1}}\left[D_{1}^{1}(z, t)\right] d z d t+\underline{\theta} \iint_{\overline{S_{1}^{1}}}\left[D_{1}^{1}(z, t)\right] d z d t \\
= & \iint_{S_{1}^{1}}\left[D_{1}^{1}(z, t)\right] d z d t-\frac{\underline{\theta}}{\underline{\theta}+\bar{\theta}} \int_{0}^{T} \int_{a}^{b}\left|D_{1}^{1}(z, t)\right| d z d t \\
\geq & 0,
\end{aligned}
$$

which contradicts the initial assumption and proves that

$$
\begin{aligned}
& N P V_{v, u}(X) \geq N P V_{v, u}(Y) \\
& \forall(v, u) \in\left(V_{1} \times U_{1}\right)\left(\gamma_{1}\right) \text { and } \forall u \in U_{1}\left(\varepsilon_{1 T}\right)
\end{aligned} \quad \Rightarrow \quad X>_{A 1 T S} Y .
$$

\section{Proof of Proposition 6}

\section{Sufficiency:}

We want to prove that

$$
\begin{array}{ll} 
& X>_{A 1 T 2 S} Y \\
\Rightarrow \quad & N P V_{v, u}(X) \geq N P V_{v, u}(Y) \\
& \forall(v, u)\left(V_{1} \times U_{2}\right)\left(\gamma_{1,2}\right), \forall u \in U_{2}\left(\varepsilon_{2 T}\right) \text { and } \forall v \in V_{1}\left(\lambda_{1 b}\right)
\end{array}
$$

Integrate the previous expression for $\Delta$ once more with respect to $z$, obtaining

$$
\begin{gathered}
\Delta=v(T)\left[\left.u^{\prime}(z) D_{1}^{2}(z, T)\right|_{a} ^{b}-\int_{a}^{b} u^{\prime \prime}(z) D_{1}^{2}(z, T) d z\right]+ \\
+\int_{0}^{T}-\left.v^{\prime}(t)\left[u^{\prime}(z) D_{1}^{2}(z, t)\right]\right|_{a} ^{b} d t-\int_{0}^{T}-v^{\prime}(t) \int_{a}^{b} u^{\prime \prime}(z) D_{1}^{2}(z, t) d z d t \geq 0 \\
v(T) u^{\prime}(b) D_{1}^{2}(b, T)+u^{\prime}(b) \int_{0}^{T}-v^{\prime}(t) D_{1}^{2}(b, t) d t- \\
-v(T) \int_{a}^{b} u^{\prime \prime}(z) D_{1}^{2}(z, T) d z+\int_{0}^{T} \int_{a}^{b}\left(-v^{\prime}(t)\right)\left(-u^{\prime \prime}(z)\right) D_{1}^{2}(z, t) d z d t \geq 0 \\
v(T) u^{\prime}(b) D_{1}^{2}(b, T)+\Lambda+\bar{E}+\bar{\Gamma} \geq 0
\end{gathered}
$$

Hence in the case of Almost First-order Time and Second-order Stochastic Dominance four elements must be non-negative. $v(T) u^{\prime}(b) D_{1}^{2}(b, T)$ must simply be non-negative. The remaining three elements must be non-negative overall, but can be partitioned into a region of violation and a region of non-violation, with three respective restrictions on the relative violation. 
Define the set of realisations where $D_{1}^{2}(b, t)<0$, for any $t$ where $z=b$ as $S_{1, b}$ and its complement as $\overline{S_{1, b}}$, so that

$$
\Lambda=u^{\prime}(b) \int_{S_{1, b}}\left(-v^{\prime}(t)\right) D_{1}^{2}(b, t) d t+u^{\prime}(b) \int_{\overline{S_{1, b}}}\left(-v^{\prime}(t)\right) D_{1}^{2}(b, t) d t .
$$

The integral over $S_{1, b}$ is negative while the integral over its complement $\overline{S_{1, b}}$ is positive. Therefore, in an analogous fashion to the proof of Proposition 5, in order for $\Lambda \geq 0$ the area where $D_{1}^{2}(b, t)<0$ must be $\lambda_{1 b}$ smaller than the total area enclosed between the two distributions, where the restriction is obtained by requiring that any discount function $v$ belong to $V_{1}\left(\lambda_{1 b}\right)$.

$\bar{E}$ is similar to $E$ in the previous proof. By restricting the utility function to belong to the subset $U_{2}\left(\varepsilon_{2 T}\right)$, we obtain the requirement that in period $T$ the area where $D_{1}^{2}(z, T)<0$ cannot be larger that $\varepsilon_{2 T}$ multiplied by the total area between the two distributions.

Moving to $\bar{\Gamma}$, define an interval of violation and its complement in the usual way:

$\bar{\Gamma}=\iint_{S_{1}^{2}}\left(-v^{\prime}(t)\right)\left(-u^{\prime \prime}(z)\right) D_{1}^{2}(z, t) d z d t+\iint_{\overline{S_{1}^{2}}}\left(-v^{\prime}(t)\right)\left(-u^{\prime \prime}(z)\right) D_{1}^{2}(z, t) d z d t$.

Again following the proof of Proposition $5 \operatorname{define} \inf _{z \in[a, b] \forall t}\left\{v^{\prime}(t) u^{\prime \prime}(z)\right\}=\underline{\vartheta}$ and $\sup _{z \in[a, b] \forall t}\left\{v^{\prime}(t) u^{\prime \prime}(z)\right\}=\bar{\vartheta}$, so that the minimum $\Omega$ is

$$
\bar{\Gamma}^{*}=\underline{\vartheta} \iint_{S_{1}^{2}} D_{1}^{2}(z, t) d z d t+\bar{\vartheta} \iint_{\overline{S_{1}^{2}}} D_{1}^{2}(z, t) d z d t .
$$

Both elements of $\bar{\Gamma}$ are relatively larger than the corresponding elements of $\bar{\Gamma}^{*}$.

We are looking for a set of preferences $\left(V_{1} \times U_{2}\right)\left(\gamma_{1,2}\right)$ for which $\bar{\Gamma}^{*} \geq 0$, which are

$$
\begin{gathered}
\sup \left[v^{\prime}(t) u^{\prime \prime}(z)\right] \leq \inf \left[v^{\prime}(t) u^{\prime \prime}(z)\right] \frac{\iint_{\overline{S_{1}^{2}}}\left[D_{1}^{2}(z, t)\right] d z d t}{\iint_{S_{1}^{2}}\left[F_{1}^{2}(z, t)-G_{1}^{2}(z, t)\right] d z d t} \\
\left.\sup \left[v^{\prime}(t) u^{\prime \prime}(z)\right] \leq \inf \left[v^{\prime}(t) u^{\prime \prime}(z)\right)\right] \frac{\iint_{S_{2}^{2}}\left[G_{1}^{2}(z, t)-F_{1}^{2}(z, t)\right] d z d t}{\iint_{S_{2}^{2}}\left[F_{1}^{2}(z, t)-G_{1}^{2}(z, t)\right] d z d t}
\end{gathered}
$$

By letting $(v, u) \in\left(V_{1} \times U_{2}\right)\left(\gamma_{1,2}\right)$, then, by definition of $\left(V_{1} \times U_{2}\right)\left(\gamma_{1,2}\right)$, we know that

$$
\left[v^{\prime}(t) u^{\prime \prime}(z)\right] \leq \sup \left[v^{\prime}(t) u^{\prime \prime}(z)\right] \leq \inf \left[v^{\prime}(t) u^{\prime \prime}(z)\right]\left[\frac{1}{\gamma_{1,2}}-1\right],
$$

which implies that $\Omega^{*} \geq 0$ holds and therefore, $N P V_{v, u}(X) \geq N P V_{v, u}(Y)$.

Necessity:

Starting from Eq. (5) and using the increasing and concave utility function defined in proving necessity in Proposition 3, the proof proceeds in just the same fashion as for Proposition 5 and is therefore omitted. 